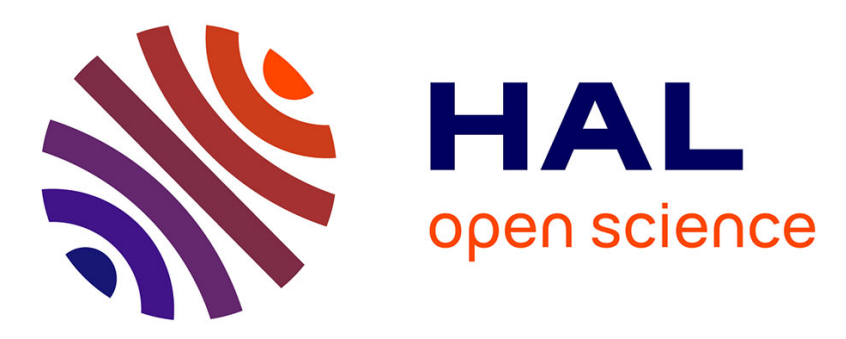

\title{
Extended complexification method to study nonlinear passive control
}

Diala Bitar, Alireza Ture Savadkoohi, Claude-Henri Lamarque, Emmanuel

Gourdon, Manuel Collet

\section{- To cite this version:}

Diala Bitar, Alireza Ture Savadkoohi, Claude-Henri Lamarque, Emmanuel Gourdon, Manuel Collet. Extended complexification method to study nonlinear passive control. Nonlinear Dynamics, 2020, 99, pp.1433-1450. 10.1007/s11071-019-05365-z . hal-02364913

\section{HAL Id: hal-02364913 https://hal.science/hal-02364913}

Submitted on 12 Jun 2020

HAL is a multi-disciplinary open access archive for the deposit and dissemination of scientific research documents, whether they are published or not. The documents may come from teaching and research institutions in France or abroad, or from public or private research centers.
L'archive ouverte pluridisciplinaire HAL, est destinée au dépôt et à la diffusion de documents scientifiques de niveau recherche, publiés ou non, émanant des établissements d'enseignement et de recherche français ou étrangers, des laboratoires publics ou privés. 


\title{
Extended complexification method to study nonlinear passive control
}

\author{
D. Bitar, A. Ture Savadkoohi, C-H. Lamarque, \\ E. Gourdon, and M. Collet
}

Nonlinear Dyn 99, 1433-1450 (2020)

\begin{abstract}
The present research work aims to design a passive vibration control based on nonlinear energy pumping. An extended asymptotic approach is introduced based on the invariant manifold approach for the case of 1:1 resonance. It consists in introducing an extended form of Manevitch's complex variables, taking into consideration higher harmonics, enabling the detection of the invariant manifold of the system at fast time scale. At the slow time scale, equilibrium points and singularities are identified analytically in order to predict periodic regimes and strongly modulated responses. The example of a passive shunt loudspeaker using a nonlinear absorber is studied. Unlike classical investigations, the first and third harmonics are taken into consideration. It is demonstrated that the presence of the third harmonic improves the approximations of the results. Different cases are considered, where the obtained analytical results are in good agreement with those obtained via direct numerical integration of the principal system of equations.
\end{abstract}

Keywords Nonlinear energy sink · Extended complexification method · Passive shunt loudspeaker · Fast/Slow dynamics.

\section{Introduction}

In order to reach desirable behaviors, researchers employed structural and acoustical systems to control noise and vibrations [1]. The structural control process covers large categories varying from active [2] to passive [3,4] controller systems. The passive control processes do not need any additional feeding systems for their activation and they endow characteristics of the main vibratory systems or coupled ones (as passive controllers) for reducing vibrations. Classical passive controllers are linear named as tuned mass dampers [5] for mechanical systems and Helmholtz resonators [6] in acoustics. These linear devices are very efficient around a narrow frequency width, but they create other resonances in the system. Some systems and oscillators perform nonlinear behaviors due to their spacial configurations and/or due to their physical properties. For example double pendulums and also micro electro-mechanical systems can present quadratic behaviors due to electrostatic excitations and piezoelectric materials $[7,8]$ while shallow arches possess both quadratic and cubic nonlinearities [7]. There have been some attempts to exploit nonlinear properties of oscillators for passive control process. Roberson [9] showed that by adding a cubic nonlinearity to a classical passive system, the cancellation band of the device increases with respect the one of the linear damper [10]. Since then many types of nonlinear absorbers are developed which as representative examples of the vast list in this domain we can name: pendulum-type absorbers [11], autoparametric absorbers [12], systems with softening springs, for instance arrays of Belleville washers mounted back to back [13], impact systems [14] and nonlinear energy sink (NES) [15]. The NES systems

D. Bitar, A. Ture Savadkoohi, C-H. Lamarque, E. Gourdon

Univ Lyon, Ecole Nationale des Travaux Publics de l'Etat (ENTPE), LTDS UMR CNRS 5513,

3 rue Maurice Audin, F-69518 Vaulx-en-Velin Cedex, France.

E-mail: alireza.turesavadkoohi@entpe.fr

D. Bitar, M. Collet

Univ Lyon, Ecole Centrale de Lyon (ECL), LTDS UMR CNRS 5513,

36 avenue Guy de Collongue, F-69134 Ecully Cedex, France. 
in their original developments consist of a pure cubic nonlinear system (with no linear part) i.e. without any linear frequency. This property permits the NES to enter into resonance with any excitations enabling the transfer of vibrational energy of main structures in a one way and irreversible manner [16,17]. Such passive systems are applied in different domain such as aerospace [18], civil engineering (buildings [19], cables [20]) and acoustics [21]. The theoretical background of such systems is quite extensive and still is in the progressing stage: one of the mostly used techniques is applying complex variables of Manevitch [22] to system and keeping first harmonics [23]. For prestressed systems, e.g. the systems in gravitational fields, in addition to first harmonics, constant terms of Fourier series should be taken into account as well [24-26]. The aim of the current paper is to include the third harmonics (in addition to the first ones) for a better prediction of the principle coupled system behaviors. The organization of the paper is as it follows: In section 2 we introduce a general representation of an electroacoustic absorber, consisting of a loudspeaker coupled to an electrical NES. In a brief way, the system is treated analytically allowing the detection of the invariant manifold. An extended version of the method is developed in section 3, taking into consideration higher harmonics. In the same section, invariant manifold of the system together with the equilibrium points and singularities specific to different dynamical regimes are identified. Section 4 is dedicated to highlight the consequences of adding higher harmonic to the analytical treatments. Two different regimes are illustrated with results obtained with direct integration of the system compared to the analytical developments. At the end of this paper conclusions and perspectives are given in section 5 .

\section{Considered model and classical treatment}

The dynamics of an electroacoustic loudspeaker, shunt to an electrical nonlinear (cubic nonlinearity) circuit and subjected to an external periodically varying sound pressure can be described by the following differential system $[27,28]$

$$
\left\{\begin{array}{l}
M_{m s} \ddot{x}(t)+R_{m s} \dot{x}(t)+C_{m c}^{-1} x(t)-C B l \dot{V}_{c}(t)=S A_{m} \cos (\omega t), \\
C\left(L_{e}+L_{c}\right) \ddot{V}_{c}(t)+C\left(R_{e}+R_{c}\right) \dot{V}_{c}(t)+k V_{c}^{3}+B l \dot{x}(t)=0,
\end{array}\right.
$$

where $x$ and $V_{c}$ describe the small displacement of the loudspeaker membrane and the electrical potential applied to the capacitor in the nonlinear shunt circuit, with $\cdot \equiv d / d t . M_{m s}, R_{m s}$ and $C_{m c}$ are the mass, the mechanical resistance of the moving bodies and the equivalent compliance of the enclosed loudspeaker. $B l$ is the force factor of the transducer, $B$ represents the magnetic field magnitude and $l$ stands for the length of the wire in the voice coil. $A_{m}$ represents the pressure amplitude, $\omega$ the angular frequency and $S$ the diaphragm area. From the electrical side, $R_{e}$ and $L_{e}$ are respectively the DC resistance and the inductance of the voice coil and $B l \dot{V}_{c}(t)$ is the back electromotive force. $R_{c}, L_{c}$ and $C$ are the inductor, resistor and capacitance of the corresponding nonlinear shunt circuit. $k$ is the nonlinear coefficient (related to the design of the electronic circuit). After introducing the following non-dimensional time variable $T=\omega_{0} t$ with $\omega_{0}=\sqrt{1 /\left(M_{m s} C_{m c}\right)}$, the scaled governing system can be written as it follows

$$
\left\{\begin{aligned}
x^{\prime \prime}+\varepsilon \lambda x^{\prime}+x-\varepsilon \alpha V_{c}^{\prime} & =\varepsilon f \cos (\Omega T), \\
\varepsilon\left(V_{c}^{\prime \prime}+\gamma V_{c}^{\prime}+\xi V_{c}^{3}+\eta x^{\prime}\right) & =0
\end{aligned}\right.
$$

where $\varepsilon=\left(L_{e}+L_{c}\right) / L_{0}$ with $L_{0}=1$ can be considered as our small dimensionless parameter, $\Omega=\omega / \omega_{0}$, $R_{m s} C_{m c} \omega_{0}=\varepsilon \lambda, C B l C_{m c} \omega_{0}=\varepsilon \alpha, S A_{m} C_{m c}=\varepsilon f,\left(R_{e}+R_{c}\right) /\left(\omega_{0} L_{0}\right)=\varepsilon \gamma, k /\left(C \omega_{0}^{2} L_{0}\right)=\varepsilon \xi, B l /\left(C \omega_{0} L_{0}\right)=$ $\varepsilon \eta$ and ${ }^{\prime} \equiv d / d T$. The choice of parameters scaling is governed by their physical range. The Slow Invariant Manifold (SIM) of the system can be obtained by treating the system (2) analytically after introducing the following Manevitch's complex variables [22,25]:

$$
\left\{\begin{array}{l}
x^{\prime}+i \Omega x=\varphi_{11}(T) e^{i \Omega T} \\
V_{c}^{\prime}+i \Omega V_{c}=\varphi_{21}(T) e^{i \Omega T}
\end{array}\right.
$$

By writing the complex variables into their polar form as $\varphi_{11}=N_{11} e^{i \delta_{11}}$ and $\varphi_{21}=N_{21} e^{i \delta_{21}}$, the invariant manifold of the scaled system (2) at the fast time scale $\tau_{0}=\varepsilon^{0} T$ can be written as follows

$$
N_{11}=\frac{N_{21}}{\eta} \sqrt{\gamma^{2}+\left(1-\frac{3}{4} \xi N_{21}^{2}\right)^{2}} .
$$


For $\alpha=\eta=0.2, \gamma=0.35, \xi=0.5, \lambda=0.1, \varepsilon=0.01$, Figure 1 shows a typical SIM with unstable zone represented in dashed line passing through local extrema of the SIM, which are clarified as

$$
\begin{aligned}
& n_{21}=\frac{2}{3} \sqrt{\frac{2}{\xi}-\frac{\sqrt{1-3 \gamma^{2}}}{\xi}} \\
& n_{22}=\frac{2}{3} \sqrt{\frac{2}{\xi}+\frac{\sqrt{1-3 \gamma^{2}}}{\xi}} .
\end{aligned}
$$

At the resonance frequency corresponding to $\Omega=\omega / \omega_{0}=1$, the scaled system (2) has been solved using a Runge-Kutta algorithm with the initial conditions $s(0)=V_{c}(0)=V_{c}^{\prime}(0)=0$ and $x^{\prime}(0)=3.7$ which corresponds to $N_{11}=3.7$ and $N_{21}=0$. Figure 1 shows a comparison between the analytical SIM which provides information about all possible behaviors of the system and the corresponding results obtained via numerical integration of System (2). Two different scenarios are shown: the first one is dedicated for an equilibrium point represented by a pink triangle (weak forcing term $f=0.2$ ) and the second one for a Strongly Modulated Response (SMR) (higher forcing term $f=0.5$ ). The numerical results oscillate around the right stable branch of the SIM then jump through bifurcation to experience the left stable branch until its amplitude decreases quasi-linearly (a) or exhibit a persistent hysteretic SMR (b). Remarkably, jump phenomenon occur around the extrema $n_{21}$ and $n_{22}$. In addition the results of the numerical integration are not symmetrically distributed around the right branch of the SIM due to the contribution of higher harmonics. So, the following section is dedicated to extend Manevitch's complex variables including higher harmonics, expecting improvement of the agreement between the numerical results and the analytical SIM.

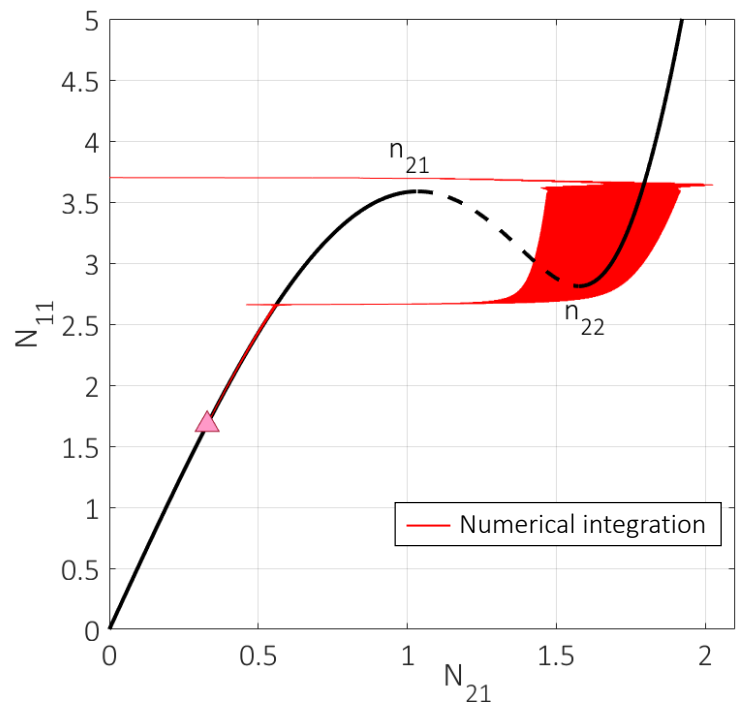

(a)

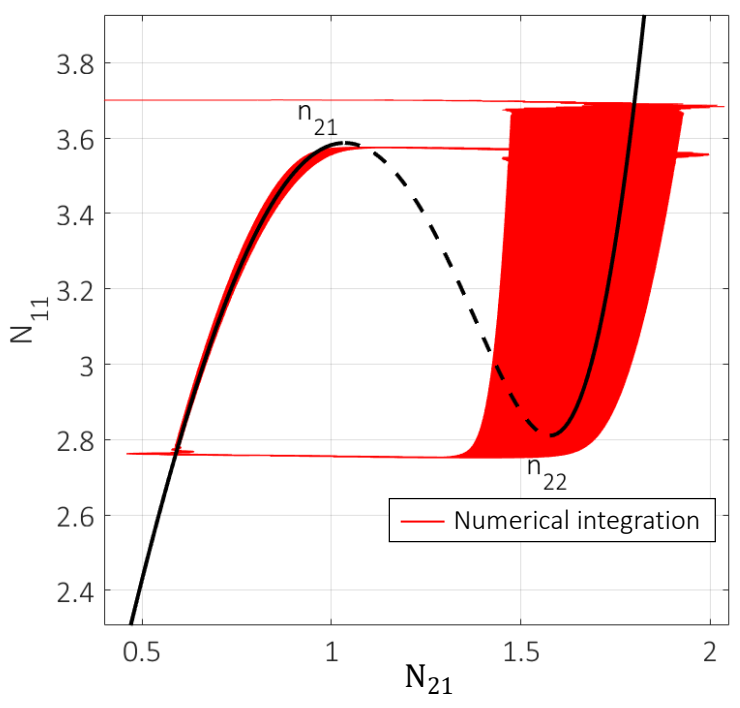

(b)

Fig. 1 The curve in black describes the Slow Invariant Manifold (SIM) of the system for $\xi=0.5$ and $\gamma=0.35$ with the dashed line illustrating the unstable zone. The numerical integration of the scaled system (2) for $\alpha=\eta=0.2, \lambda=0.1$ and $\varepsilon=0.01$ under forced oscillations are represented in red. (a) and (b) correspond to a periodic regime with weak force $f=0.2$ and a strongly modulated response with higher forcing term $f=0.5$ respectively [29].

\section{Extension of the Method}

The analytical results obtained by applying classical form of Manevitch's complex variables [22] requires an extension of the method by taking into consideration higher harmonics. Consequently, we consider the following scaled 2 dofs system, which consists of a primary linear system with the mass 1 and the coupled NES with the mass $\varepsilon, 0<\varepsilon<<1$ 


$$
\left\{\begin{array}{c}
x_{1}^{\prime \prime}+x_{1}+\varepsilon k_{l 1}\left(x_{1}, x_{1}^{\prime}, x_{2}, x_{2}^{\prime}\right)+\varepsilon k_{n 1}\left(x_{1}, x_{1}^{\prime}, x_{2}, x_{2}^{\prime}\right)=\varepsilon f \cos (\Omega T) \\
\varepsilon\left(x_{2}^{\prime \prime}+k_{l 2}\left(x_{1}, x_{1}^{\prime}, x_{2}, x_{2}^{\prime}\right)+k_{n 2}\left(x_{1}, x_{1}^{\prime}, x_{2}, x_{2}^{\prime}\right)\right)=0
\end{array}\right.
$$

$x_{1}$ and $x_{2}$ represent the displacement of the primary system and the NES respectively and $f$ is the external forcing term. The linear and nonlinear functions are characterized by $k_{l j}$ and $k_{n j}$ respectively with $j \in\{1,2\}$. The main system is excited around its resonance frequency 1 , with $\Omega=1+\varepsilon \sigma$ where $\sigma$ is the detuning parameter. Then, we introduce an extended form of Manevitch's complex variables [30]

$$
x_{j}^{\prime}+i \Omega x_{j}=\sum_{m=1}^{M_{j}} \varphi_{j m} e^{i m \Omega T} \quad \text { for } j=1,2
$$

which yields to:

$$
\left\{\begin{array}{l}
x_{j}^{\prime}=\frac{1}{2} \sum_{m=1}^{M_{j}}\left(\varphi_{j m} e^{i m \Omega T}+\text { c.c. }\right) \\
x_{j}=\frac{1}{2 i \Omega} \sum_{m=1}^{M_{j}}\left(\varphi_{j m} e^{i m \Omega T}-\text { c.c. }\right)
\end{array}\right.
$$

where c.c. stands for the complex conjugate of the rest of the terms in the equation. The derivation of Equation (7) yields

$$
x_{j}^{\prime \prime}+i \Omega x_{j}^{\prime}=\sum_{m=1}^{M_{j}}\left(\varphi_{j m}^{\prime}+i \Omega m \varphi_{j m}\right) e^{i m \Omega T} .
$$

Then, after replacing Equation (8) into Equation (9) we obtain:

$$
x_{j}^{\prime \prime}=\sum_{m=1}^{M_{j}}\left[\left(\varphi_{j m}^{\prime}+i \Omega\left(m-\frac{1}{2}\right) \varphi_{j m}\right) e^{i m \Omega T}-\frac{i \Omega}{2} \varphi_{j m}^{*} e^{-i m \Omega T}\right] .
$$

In order to detect the analytical invariant, we study in the following section the system behavior around the 1:1 resonance using a multiple scales approach [31].

\subsection{Multiple scale analysis of the system}

Analyzing the system at different time scales, we introduce the fast time $\tau_{0}$ and slow times $\tau_{1}, \tau_{2}, \ldots$ as $T=\tau_{0}, \tau_{1}=\varepsilon \tau_{0}, \tau_{2}=\varepsilon^{2} \tau_{0} \ldots$ so,

$$
\varphi_{j m}^{\prime}=\frac{\partial \varphi_{j m}}{\partial \tau_{0}}+\varepsilon \frac{\partial \varphi_{j m}}{\partial \tau_{1}}+\ldots \quad \text { for } j \in\{1,2\}
$$

It should be mentioned that the $\varepsilon$ parameter defined in different time scale, is a physical parameter, i.e. mass ratio of the two oscillators and not a bookkeeping parameter which never tends to zero. This means that for treatment of described system in Eq. 2 or Eq. 6 we do not need to use singular perturbation methods [32] for revealing fast/slow dynamics of the system [33]. Replacing the generalized complex variables (10), (8) and (11) in the scaled system (6) we get the following system at the first order 


$$
\left\{\begin{array}{c}
\varepsilon^{0}\left[\sum_{m=1}^{M_{1}}\left(\frac{\partial \varphi_{1 m}}{\partial \tau_{0}}+i(m-1) \varphi_{1 m}\right) e^{i m \Omega \tau_{0}}\right] \\
+\varepsilon^{1}\left[\sum_{m=1}^{M_{1}}\left(\left(\frac{\partial \varphi_{1 m}}{\partial \tau_{1}}+i m \sigma \varphi_{1 m}\right) e^{i m \Omega \tau_{0}}-i \sigma \varphi_{1 m}^{*} e^{-i m \Omega \tau_{0}}\right)-\frac{1}{2} f\left(e^{i \Omega \tau_{0}}-e^{-i \Omega \tau_{0}}\right)\right. \\
\quad+K_{l 1}\left(\varphi_{11}, \ldots, \varphi_{1 M_{1}}, \varphi_{21}, \ldots, \varphi_{1 M_{2}}, \varphi_{11}^{*}, \ldots, \varphi_{1 M_{1}}^{*}, \varphi_{21}^{*}, \ldots, \varphi_{1 M_{2}}^{*}\right) \\
\left.\quad+K_{n 1}\left(\varphi_{11}, \ldots, \varphi_{1 M_{1}}, \varphi_{21}, \ldots, \varphi_{1 M_{2}}, \varphi_{11}^{*}, \ldots, \varphi_{1 M_{1}}^{*}, \varphi_{21}^{*}, \ldots, \varphi_{1 M_{2}}^{*}\right)\right]+\cdots=0 \\
\varepsilon^{0}\left[\sum_{m=1}^{M_{2}}\left(\left(\frac{\partial \varphi_{2 m}}{\partial \tau_{0}}+i\left(m-\frac{1}{2}\right) \varphi_{2 m}\right) e^{i m \Omega \tau_{0}}-\frac{i}{2} \varphi_{2 m}^{*} e^{-i m \Omega \tau_{0}}\right)\right. \\
\quad+K_{l 2}\left(\varphi_{11}, \ldots, \varphi_{1 M_{1}}, \varphi_{21}, \ldots, \varphi_{1 M_{2}}, \varphi_{11}^{*}, \ldots, \varphi_{1 M_{1}}^{*}, \varphi_{21}^{*}, \ldots, \varphi_{1 M_{2}}^{*}\right) \\
\left.\quad+K_{n 2}\left(\varphi_{11}, \ldots, \varphi_{1 M_{1}}, \varphi_{21}, \ldots, \varphi_{1 M_{2}}, \varphi_{11}^{*}, \ldots, \varphi_{1 M_{1}}^{*}, \varphi_{21}^{*}, \ldots, \varphi_{1 M_{2}}^{*}\right)\right]+\cdots=0
\end{array}\right.
$$

where $K_{l j}$ and $K_{n j}$ with $j \in\{1,2\}$ are the $\varepsilon^{0}$ orders of the linear and nonlinear operators $k_{l j}$ and $k_{n j}$ respectively. We endow Galerkin's technique $[8,34,35]$ in order to keep the $m^{\text {th }}$ harmonics and truncate other ones. For instance, for a general function $\mathscr{Q}\left(\tau_{0}, \tau_{1}, \tau_{2}, \ldots\right)$ the $m^{\text {th }}$ harmonic will be kept via:

$$
\mathscr{P}\left(\tau_{1}, \tau_{2}, \ldots\right)=\frac{\Omega}{2 \pi} \int_{0}^{2 \pi / \Omega} \mathscr{Q}\left(\varphi_{11}, \ldots, \varphi_{1 M_{1}}, \varphi_{21}, \ldots, \varphi_{2 M_{2}}, \tau_{1}, \tau_{2}, \ldots\right) e^{-i m \Omega \tau_{0}} d \tau_{0}, \quad \text { with } m \in \mathbb{N}_{0}
$$

In using the Equation (13), for our developments we suppose that $\varphi_{1 m}$ and $\varphi_{2 n}$ with $m \in\left\{1, \ldots, M_{1}\right\}$ and $n \in\left\{1, \ldots, M_{2}\right\}$ are independent of time $\tau_{0}$. This assumption will be verified during multiple scale method or when we seek for asymptotic response when $\tau_{0} \rightarrow \infty$. The present methodology enables the detection of the system invariant at the fast time scale $\tau_{0}$, together with the detection of the system behaviors at slower time scales.

\subsubsection{The system behavior at $\tau_{0}$ time scale}

First equation of the System (12) at the order $\varepsilon^{0}$ yields to:

$$
\left\{\begin{array}{l}
\text { for } m=1, \quad \frac{\partial \varphi_{11}}{\partial \tau_{0}}=0 \\
\text { for } m>1, \quad \frac{\partial \varphi_{1 m}}{\partial \tau_{0}}+i(m-1) \varphi_{1 m}=0
\end{array}\right.
$$

then,

$$
\left\{\begin{array}{l}
\text { for } m=1, \quad \varphi_{11}=\varphi_{11}\left(\tau_{1}, \tau_{2}, \ldots\right) \\
\text { for } m>1, \quad \varphi_{1 m}=C_{m} e^{-i(m-1) \tau_{0}}, \quad C_{m} \text { are constants of integration. }
\end{array}\right.
$$

However, the second equation in System (7) at the order $\varepsilon^{0}$ can be expressed as in the following form

$$
\mathscr{H}=\left\{\begin{aligned}
\frac{\partial \varphi_{21}}{\partial \tau_{0}}= & H_{1}\left(\varphi_{11}, \varphi_{11}^{*}, \ldots, \varphi_{1 M_{1}}, \varphi_{1 M_{1}}^{*}, \varphi_{21}, \varphi_{21}^{*}, \ldots, \varphi_{2 M_{2}}, \varphi_{2 M_{2}}^{*}\right), \\
& \vdots \\
\frac{\partial \varphi_{2 m}}{\partial \tau_{0}}= & H_{m}\left(\varphi_{11}, \varphi_{11}^{*}, \ldots, \varphi_{1 M_{1}}, \varphi_{1 M_{1}}^{*}, \varphi_{21}, \varphi_{21}^{*}, \ldots, \varphi_{2 M_{2}}, \varphi_{2 M_{2}}^{*}\right), \\
& \vdots \\
\frac{\partial \varphi_{2 M_{2}}}{\partial \tau_{0}} & =H_{M_{2}}\left(\varphi_{11}, \varphi_{11}^{*}, \ldots, \varphi_{1 M_{1}}, \varphi_{1 M_{1}}^{*}, \varphi_{21}, \varphi_{21}^{*}, \ldots, \varphi_{2 M_{2}}, \varphi_{2 M_{2}}^{*}\right) .
\end{aligned}\right.
$$


System (16) is used to trace an asymptotic equilibrium governed by a SIM. Fixed points of the system are obtained when the following criterion is satisfied

$$
H_{m}\left(\varphi_{11}, \varphi_{11}^{*}, \ldots, \varphi_{1 M_{1}}, \varphi_{1 M_{1}}^{*}, \varphi_{21}, \varphi_{21}^{*}, \ldots, \varphi_{2 M_{2}}, \varphi_{2 M_{2}}^{*}\right)=0 \text { for } m=1, \ldots, M_{2}
$$

By writing the complex variables in the polar form as $\varphi_{j m}=N_{j m} e^{i \delta_{j m}}$ with $j=1,2$ and $m=1, \ldots, M_{j}$, system (17) can be re-written and reduced after separating its real and imaginary parts to the following form

$$
\mathscr{H}=\left(\begin{array}{c}
h_{r 1}\left(\delta_{11}, N_{11}, \ldots, \delta_{1 M_{1}}, N_{1 M_{1}}, \delta_{21}, N_{21}, \ldots, \delta_{2 M_{2}}, N_{2 M_{2}}\right) \\
h_{i 1}\left(\delta_{11}, N_{11}, \ldots, \delta_{1 M_{1}}, N_{1 M_{1}}, \delta_{21}, N_{21}, \ldots, \delta_{2 M_{2}}, N_{2 M_{2}}\right) \\
\vdots \\
h_{r M_{2}}\left(\delta_{11}, N_{11}, \ldots, \delta_{1 M_{1}}, N_{1 M_{1}}, \delta_{21}, N_{21}, \ldots, \delta_{2 M_{2}}, N_{2 M_{2}}\right) \\
h_{i M_{2}}\left(\delta_{11}, N_{11}, \ldots, \delta_{1 M_{1}}, N_{1 M_{1}}, \delta_{21}, N_{21}, \ldots, \delta_{2 M_{2}}, N_{2 M_{2}}\right)
\end{array}\right)=\left(\begin{array}{c}
0 \\
0 \\
\vdots \\
0 \\
0
\end{array}\right)
$$

\subsubsection{The system behavior at $\tau_{1}$ time scale}

In order to identify the possible behaviors that the system may achieve (periodic or quasi-periodic), equilibrium points and singularities should be detected by studying the system at slow time around the SIM. The first equation of System (12) at $\varepsilon^{1}$ order reads:

$$
\frac{\partial \varphi_{1 m}}{\partial \tau_{1}}=F_{1}\left(\varphi_{11}, \varphi_{11}^{*}, \ldots, \varphi_{1 M_{1}}, \varphi_{1 M_{1}}^{*}, \varphi_{21}, \varphi_{21}^{*}, \ldots, \varphi_{2 M_{2}}, \varphi_{2 M_{2}}^{*}\right) \quad \text { for } \quad m=1, \ldots, M_{1}
$$

Equations (19) can be presented as:

$$
\left\{\begin{aligned}
\frac{\partial \delta_{11}}{\partial \tau_{1}} & =f_{\delta 1}\left(\delta_{11}, N_{11}, \ldots, \delta_{1 M_{1}}, N_{1 M_{1}}, \delta_{21}, N_{21}, \ldots, \delta_{2 M_{2}}, N_{2 M_{2}}\right) \\
\frac{\partial N_{11}}{\partial \tau_{1}} & =f_{N 1}\left(\delta_{11}, N_{11}, \ldots, \delta_{1 M_{1}}, N_{1 M_{1}}, \delta_{21}, N_{21}, \ldots, \delta_{2 M_{2}}, N_{2 M_{2}}\right) \\
& \vdots \\
\frac{\partial \delta_{1 M_{1}}}{\partial \tau_{1}} & =f_{\delta M_{1}}\left(\delta_{11}, N_{11}, \ldots, \delta_{1 M_{1}}, N_{1 M_{1}}, \delta_{21}, N_{21}, \ldots, \delta_{2 M_{2}}, N_{2 M_{2}}\right) \\
\frac{\partial N_{1 M_{1}}}{\partial \tau_{1}} & =f_{N M_{1}}\left(\delta_{11}, N_{11}, \ldots, \delta_{1 M_{1}}, N_{1 M_{1}}, \delta_{21}, N_{21}, \ldots, \delta_{2 M_{2}}, N_{2 M_{2}}\right)
\end{aligned}\right.
$$

Examining the dynamical behavior of the system around the SIM leads to $\frac{\partial \mathscr{H}}{\partial \tau_{1}}=0$. Combining Systems (18) and (20) with this later relation leads to the following system

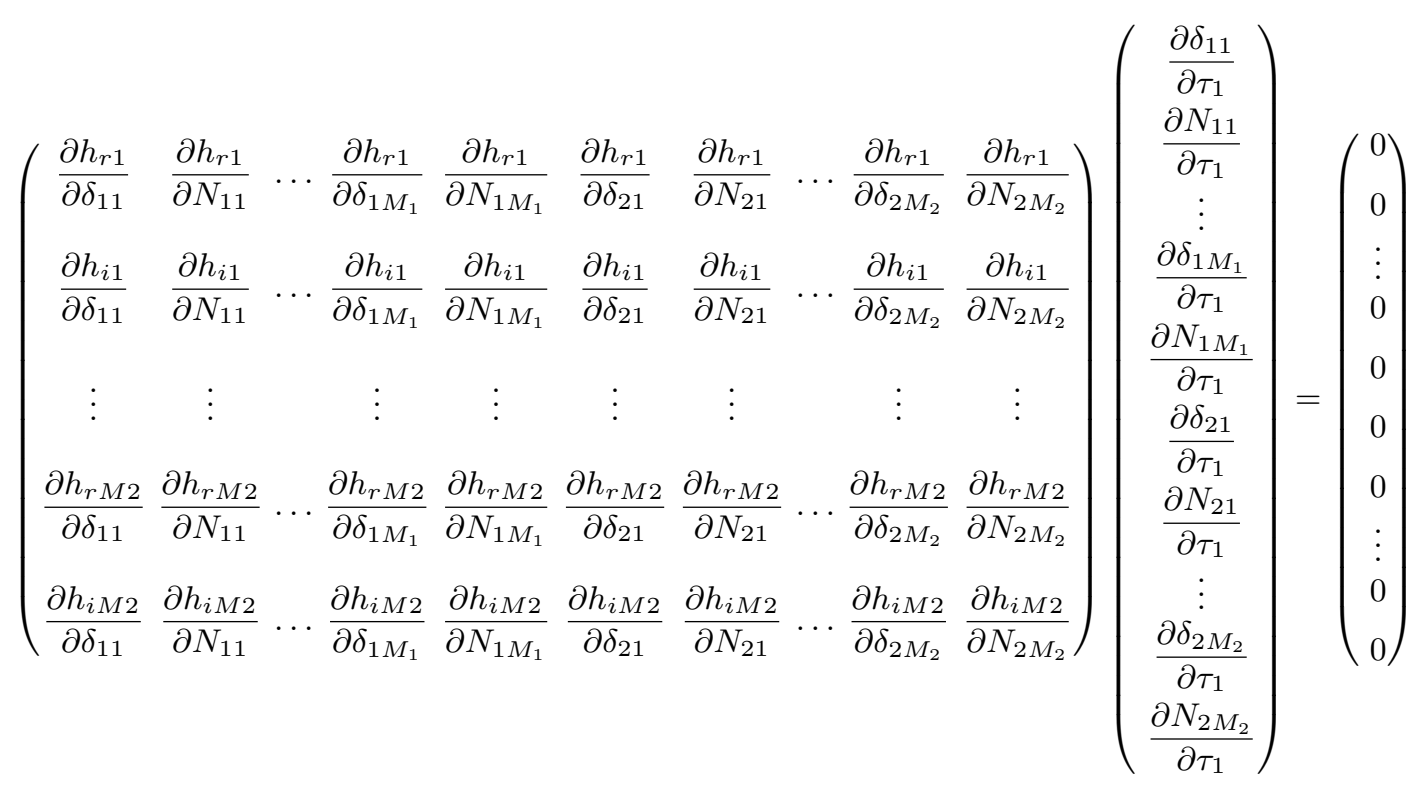


which can be written in the following form

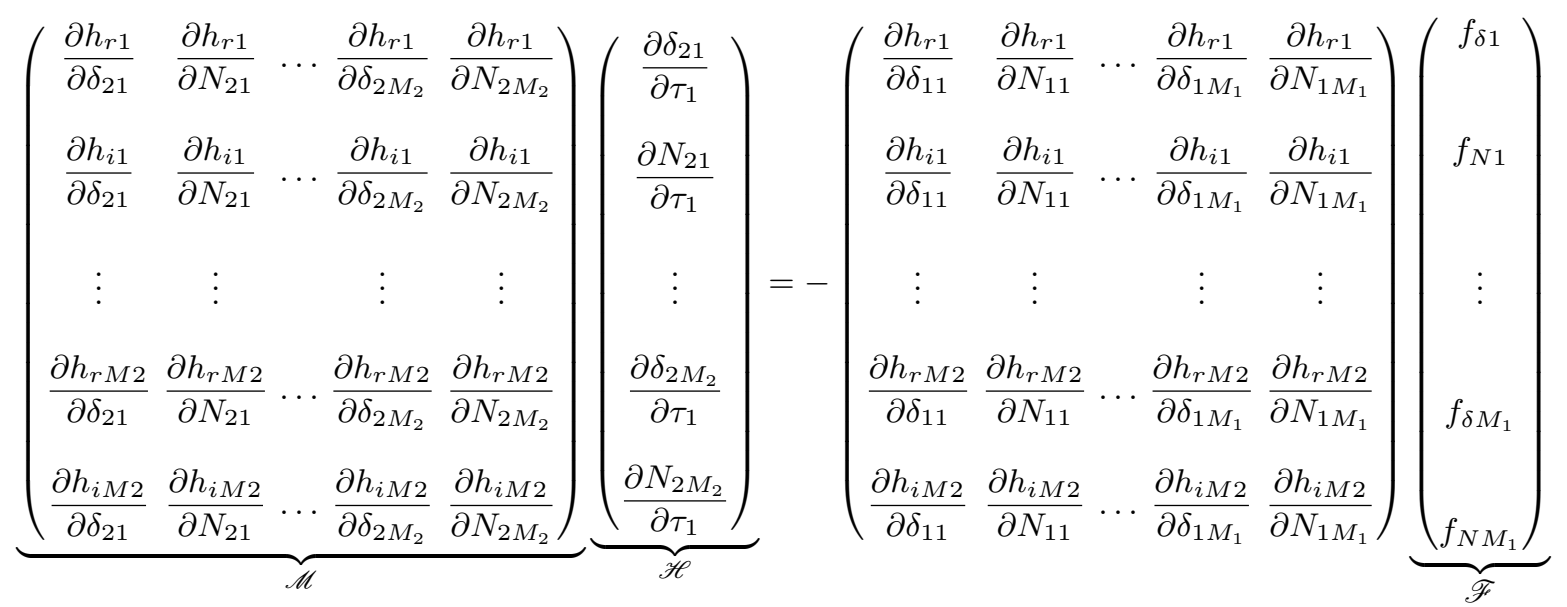

Equilibrium points of the system, can be obtained by solving the following system [36]

$$
\left\{\begin{array}{l}
\mathscr{H}=0 \\
\mathscr{F}=0 \\
\operatorname{det}(\mathscr{M}) \neq 0,
\end{array}\right.
$$

and singularities are reached when

$$
\left\{\begin{array}{l}
\mathscr{H}=0 \\
\mathscr{F}=0 \\
\operatorname{det}(\mathscr{M})=0 .
\end{array}\right.
$$

\section{Application}

In this section, we apply the extended version of Manevitch's complex variables to the problem presented in Section 2 and we use the methodology explained in Section 3 for tracing system responses. Before doing so and in order to optimize the number of equations to solve, let us analyze the contribution of number of harmonics into the system's behaviors. For this purpose, we plot in Figure 2 the comparison of $d x / d T$ and $d V_{c} / d T$ between the numerical integration, the contribution of the first harmonic only and the combination of both first and third harmonics.

According to the results obtained in Figure 2 (a) the contribution of the third harmonic of $d x / d T$ in the transient regime is negligible. Then, we can deduce that the first harmonic is sufficient to describe the dynamics of the main system. However, for $d V_{c} / d T$, the contribution of the third harmonic till $T=500$ (see Figures (b)) managed to provide more reasonable results in comparison to the the first harmonic truncation. This situation illustrated in this example is in fact quite generic. Therefore, we apply the extended version of Manevitch's complex variables, taking into account the first and third harmonics for the NES only. Let us consider in Equation (7) $M_{1}=1$ and $M_{2}=3$ with $\varphi_{22}=0$ and

$$
\left\{\begin{array} { l } 
{ x _ { 1 } = x } \\
{ x _ { 2 } = V _ { c } }
\end{array} \Longrightarrow \left\{\begin{array} { l } 
{ k _ { l 1 } ( x _ { 1 } , x _ { 1 } ^ { \prime } , x _ { 2 } , x _ { 2 } ^ { \prime } ) = \lambda x _ { 1 } ^ { \prime } - \alpha x _ { 2 } ^ { \prime } } \\
{ k _ { n 1 } ( x _ { 1 } , x _ { 1 } ^ { \prime } , x _ { 2 } , x _ { 2 } ^ { \prime } ) = 0 }
\end{array} \quad \text { and } \quad \left\{\begin{array}{l}
k_{l 2}\left(x_{1}, x_{1}^{\prime}, x_{2}, x_{2}^{\prime}\right)=\gamma x_{2}^{\prime}+\eta x_{1}^{\prime} \\
k_{n 2}\left(x_{1}, x_{1}^{\prime}, x_{2}, x_{2}^{\prime}\right)=\xi x_{2}^{3} .
\end{array}\right.\right.\right.
$$

Then, the applied new complex variables are given as follows

$$
\left\{\begin{array}{l}
x^{\prime}+i \Omega x=\varphi_{11}(T) e^{i \Omega T} \\
V_{c}^{\prime}+i \Omega V_{c}=\varphi_{21}(T) e^{i \Omega T}+\varphi_{23}(T) e^{3 i \Omega T}
\end{array}\right.
$$

For the present case, replacing the complex variables depicted in System (26) into the scaled System (2) we obtain the following equations 

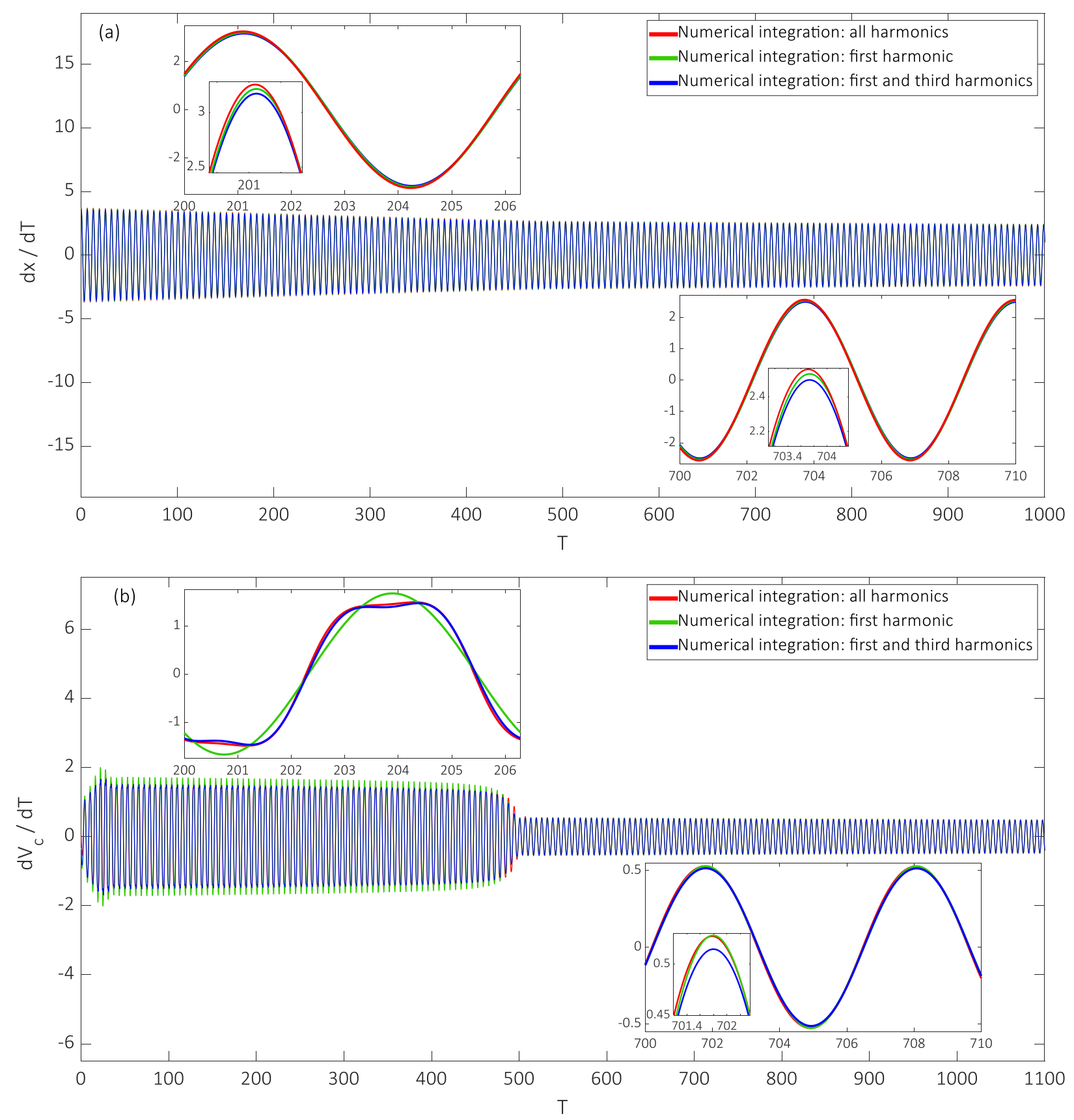

Fig. 2 A comparison between the numerical integration including all harmonic of the scaled system (2) using an explicit Runge-Kutta $(4,5)$ formula implemented in Matlab@ [37] of $d x / d T$ and $d V_{c} / d T$ (red line), their first harmonic filtering (green curve) and the combination of their first and third harmonics (blue line) for the following scaled parameters: $\alpha=\eta=0.2$, $\gamma=0.35, \xi=0.5, \lambda=0.1$ and $\varepsilon=0.01$ with the external forcing term $f=0.3$ and the detuning parameter $\sigma=0$.

$$
\left\{\begin{array}{l}
\varepsilon^{0}\left[\frac{\partial \varphi_{11}}{\partial \tau_{0}} e^{i \Omega \tau_{0}}\right]+\varepsilon^{1}\left[\left(\frac{\partial \varphi_{11}}{\partial \tau_{1}}+\sigma \varphi_{11}+\frac{\lambda}{2} \varphi_{11}-\frac{\alpha}{2} \varphi_{21}-\frac{1}{2} f\right) e^{i \Omega \tau_{0}}+\ldots\right]+\cdots=0 \\
\varepsilon^{0}\left[\left(\frac{\partial \varphi_{21}}{\partial \tau_{0}}+\frac{i}{2} \varphi_{21}+\frac{\gamma}{2} \varphi_{21}+\frac{\eta}{2} \varphi_{11}-\frac{3 i}{8} \xi\left(\varphi_{21}\left|\varphi_{21}\right|^{2}-\varphi_{23} \varphi_{21}^{*^{2}}+2 \varphi_{21}\left|\varphi_{23}\right|^{2}\right)\right) e^{i \Omega \tau_{0}}\right. \\
\left.+\left(\frac{\partial \varphi_{23}}{\partial \tau_{0}}+\frac{5 i}{2} \varphi_{23}+\frac{\gamma}{2} \varphi_{23}-\frac{i}{8} \xi\left(3 \varphi_{23}\left|\varphi_{23}\right|^{2}-\varphi_{21}^{3}+6 \varphi_{23}\left|\varphi_{21}\right|^{2}\right)\right) e^{3 i \Omega \tau_{0}}+\ldots\right]+\cdots=0
\end{array}\right.
$$


Applying the Galerkin Technique given in Equation (13) together with the multiple scales approach represented in Section 3.1 we will analyze below the system behavior around its 1:1 resonance.

4.1 The system behavior at $\tau_{0}$ time scale

Resonant terms at $\tau_{0}$ scale in system (27) yields (See Equation (14) for $m=1$ )

$$
\frac{\partial \varphi_{11}}{\partial \tau_{0}}=0 \Rightarrow \varphi_{11}=\varphi_{11}\left(\tau_{1}, \tau_{2}, \ldots\right)
$$

In addition, fixed points of the system can be described by the following system when $\lim _{\tau_{0} \rightarrow \infty} \frac{\partial \varphi_{2 m}}{\partial \tau_{0}}=0$ for $m=\{1,3\}$ (See System (16) with $M_{1}=1, M_{2}=3$ with $\varphi_{22}=0$ )

$$
\left\{\begin{array}{l}
\frac{\partial \varphi_{21}}{\partial \tau_{0}}=H_{1}\left(\varphi_{11}, \varphi_{11}^{*}, \varphi_{21}, \varphi_{21}^{*}, \varphi_{23}, \varphi_{23}^{*}\right) \\
\frac{\partial \varphi_{23}}{\partial \tau_{0}}=H_{2}\left(\varphi_{11}, \varphi_{11}^{*}, \varphi_{21}, \varphi_{21}^{*}, \varphi_{23}, \varphi_{23}^{*}\right)
\end{array}\right.
$$

Then, previous System (29) presents an asymptotic equilibrium governed by the new SIM

$$
\left\{\begin{array}{l}
H_{1}\left(\varphi_{11}, \varphi_{11}^{*}, \varphi_{21}, \varphi_{21}^{*}, \varphi_{23}, \varphi_{23}^{*}\right)=0 \\
H_{2}\left(\varphi_{11}, \varphi_{11}^{*}, \varphi_{21}, \varphi_{21}^{*}, \varphi_{23}, \varphi_{23}^{*}\right)=0
\end{array}\right.
$$

Writing the complex variables into their polar form as $\varphi_{j m}=N_{j m} e^{i \delta_{j m}}$, so system (30) can be re-written and reduced after separating its real and imaginary parts into the following form (See System (18))

$$
\mathscr{H}\left(\delta_{11}, N_{11}, \delta_{21}, N_{21}, \delta_{23}, N_{23}\right)=\left(\begin{array}{l}
h_{r 1}\left(\delta_{11}, N_{11}, \delta_{21}, N_{21}, \delta_{23}, N_{23}\right) \\
h_{i 1}\left(\delta_{11}, N_{11}, \delta_{21}, N_{21}, \delta_{23}, N_{23}\right) \\
h_{r 3}\left(\delta_{11}, N_{11}, \delta_{21}, N_{21}, \delta_{23}, N_{23}\right) \\
h_{i 3}\left(\delta_{11}, N_{11}, \delta_{21}, N_{21}, \delta_{23}, N_{23}\right)
\end{array}\right)=\left(\begin{array}{l}
0 \\
0 \\
0 \\
0
\end{array}\right)
$$

where

$$
\left\{\begin{array}{l}
h_{r 1}=N_{21}\left[\gamma+\frac{3}{4} \xi \sin \left(3 \delta_{21}-\delta_{23}\right) N_{21} N_{23}\right]+\eta \cos \left(\delta_{11}-\delta_{21}\right) N_{11}=0 \\
h_{i 1}=N_{21}\left[1-\frac{3}{4} \xi N_{21}^{2}-\frac{3}{2} \xi N_{23}^{2}+\frac{3}{4} \xi \cos \left(3 \delta_{21}-\delta_{23}\right) N_{21} N_{23}\right]+\eta \sin \left(\delta_{11}-\delta_{21}\right) N_{11}=0 \\
h_{r 3}=4 \gamma N_{23}-\xi \sin \left(3 \delta_{21}-\delta_{23}\right) N_{21}^{3}=0 \\
h_{i 3}=4 N_{23}\left[-5+\frac{3}{2} \xi N_{21}^{2}+\frac{3}{4} \xi N_{23}^{2}\right]-\xi \cos \left(3 \delta_{21}-\delta_{23}\right) N_{21}^{3}=0
\end{array}\right.
$$

Replacing Equations (34) and (35) into Equations (32) and (33) respectively we obtain

$$
\left\{\begin{array}{l}
-\gamma N_{21}-3 \gamma \frac{N_{23}^{2}}{N_{21}}=\eta \cos \left(\delta_{11}-\delta_{21}\right) N_{11} \\
N_{21}\left(1-\frac{3}{4} \xi N_{21}^{2}-15 \frac{N_{23}^{2}}{N_{21}^{2}}+3 \xi N_{23}^{2}+\frac{9}{4} \xi \frac{N_{23}^{4}}{N_{21}^{2}}\right)=\eta \sin \left(\delta_{11}-\delta_{21}\right) N_{11}
\end{array}\right.
$$

Squaring and summing Equations (36) and (37), the SIM describing the system behaviors at the infinity time scale $\tau_{0}$ can be expressed as follow

$$
\left\{\begin{aligned}
N_{11} & =\frac{N_{21}}{\eta} \sqrt{\gamma^{2}\left(1+3 \frac{N_{23}^{2}}{N_{21}^{2}}\right)^{2}+\left(1-\frac{3}{4} \xi N_{21}^{2}-15 \frac{N_{23}^{2}}{N_{21}^{2}}+3 \xi N_{23}^{2}+\frac{9}{4} \xi \frac{N_{23}^{4}}{N_{21}^{2}}\right)^{2}}, \\
\xi^{2} N_{21}^{6} & =16 N_{23}^{2}\left[\gamma^{2}+\left(-5+\frac{3}{2} \xi N_{21}^{2}+\frac{3}{4} \xi N_{23}^{2}\right)^{2}\right] .
\end{aligned}\right.
$$

The relationship between the modulus $N_{21}$ and $N_{23}$ given in Equation (38) can be expressed by the following cubic equation after setting $Z=N_{23}^{2}$ 


$$
a_{3} Z^{3}+a_{2} Z^{2}+a_{1} Z+a_{0}=0
$$

with

$$
\left\{\begin{array}{l}
a_{3}=9 \xi^{2} \\
a_{2}=-120 \xi+36 \xi^{2} N_{21}^{2} \\
a_{1}=400+16 \gamma^{2}-240 \xi N_{21}^{2}+36 \xi^{2} N_{21}^{4} \\
a_{0}=-\xi^{2} N_{21}^{6} .
\end{array}\right.
$$

Using Cardano's method, the algebraic Equation (39) can be solved analytically following the steps below. Writing $Z=\mathfrak{z}-a_{2} /\left(3 a_{3}\right)$, Equation (39) can be written as

$$
\mathfrak{z}^{3}+p \mathfrak{z}+q=0
$$

with

$$
\left\{\begin{array}{l}
p=-\frac{1}{3} \frac{a_{2}^{2}}{a_{3}^{2}}+\frac{a_{1}}{a_{3}} \\
q=\frac{1}{27} \frac{a_{2}}{a_{3}}\left(2 \frac{a_{2}^{2}}{a_{3}^{2}}-9 \frac{a_{1}}{a_{3}}\right)+\frac{a_{0}}{a_{3}} .
\end{array}\right.
$$

The discriminant $\Delta_{\text {card }}$ of the cubic Equation (41) is given by

$$
\Delta_{\text {card }}=-\left(4 p^{3}+27 q^{2}\right)
$$

According to the values of $\gamma$ and $\xi$ the sign of $\Delta_{\text {card }}$ changes. Then, the existence of a single and multiple real roots for $N_{23}$ in terms of $N_{21}$ varies. Consequently, for a given couple of parameters $(\gamma, \xi)$ the solutions can be detailed as follows

$-\Delta_{\text {card }} \leq 0, N_{23}$ has a unique solution for $0<N_{23} \leq R_{1}$ and $N_{23} \neq R_{2}$,

$-\Delta_{\text {card }}>0, N_{23}$ has a unique solution for $R_{1}<N_{23} \leq R_{2}$.

Therefore, the SIM can be easily represented in two dimensional manner as $N_{21}$ versus $N_{23}$. In order to study the influence of adding the third harmonic to classical version of Manevitch's complex variables, we consider the following parameters: $\varepsilon=0.01, \eta=\alpha=0.2, \gamma=0.35, \lambda=0.1$ and $\xi=0.5$. For the system with the following initial conditions (Corresponding to the initial velocity of the membrane)

$$
\begin{cases}x(0)=0 & V_{c}(0)=0 \\ \frac{d x}{d T}=v_{0} & \frac{d V_{c}}{d T}=0\end{cases}
$$

which corresponds to

$$
\left\{\begin{array}{l}
N_{11}(0)=v_{0} \\
N_{21}(0)=0 \\
N_{23}(0)=0
\end{array}\right.
$$

Figure 3 shows a 3D flow of the SIM with the first harmonics view $\left(N_{11}\right.$ vs $\left.N_{21}\right)$. First of all, we can notice that the geometry of the SIM (based on the first and third harmonics) changes for higher amplitudes with the occurrence of three values of $N_{11}$ versus $N_{21}$. Figure 4 shows a comparison between the analytical classical SIM (black thick curve) based on first harmonics i.e. terms of $\varphi_{n 1} e^{i \Omega T}$ in Equation (26) and modified SIM (green triangles) obtained after considering the third harmonics for the NES variables, the numerical results obtained by a direct integration of the system (2) (red line). One can clearly see that the addition of the third harmonic managed to decrease the gap between the analytical approximations and the numerical results, especially during jump phenomenon. For $\Delta_{\text {card }}<0$, Equation (41) has one real root and the solutions are expressed as follows

$$
\mathfrak{z}_{0}=u_{0}^{\frac{1}{3}}+v_{0}^{\frac{1}{3}}
$$




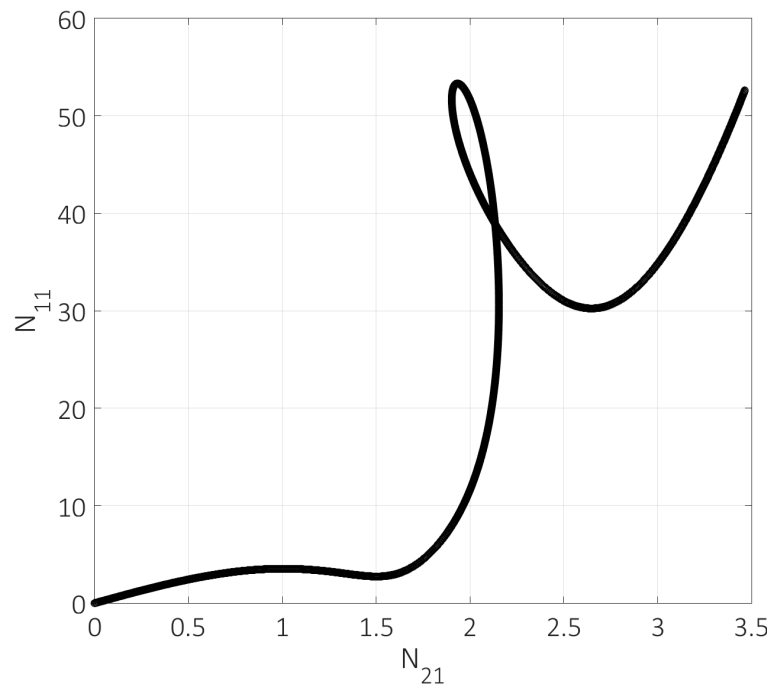

(a)

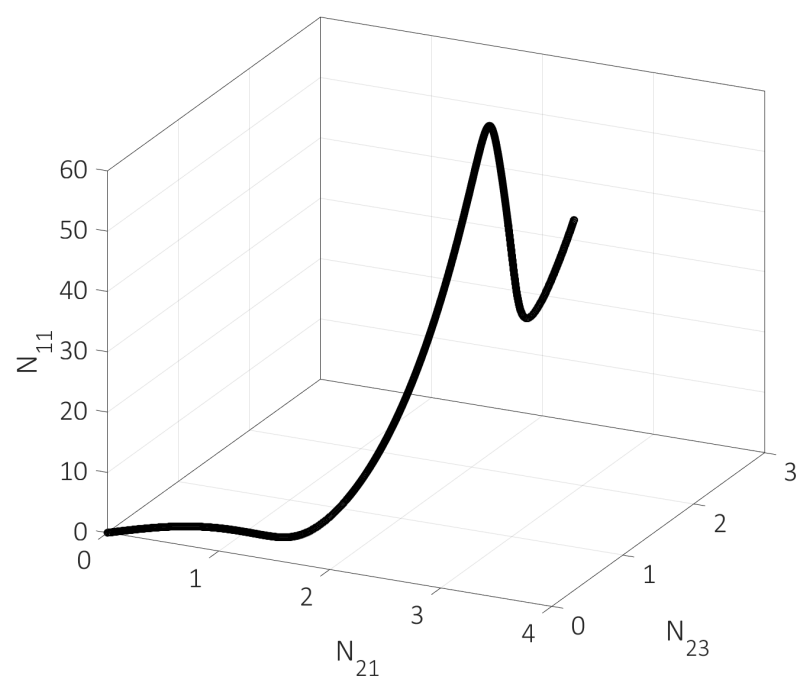

(b)

Fig. 3 (a) 2 D flow of the SIM $N_{11}$ vs $N_{21}$, (b) 3 D flow of the SIM $N_{11}$ vs $N_{21}$ and $N_{23}$ for $\xi=0.5$ and $\eta=0.2$.

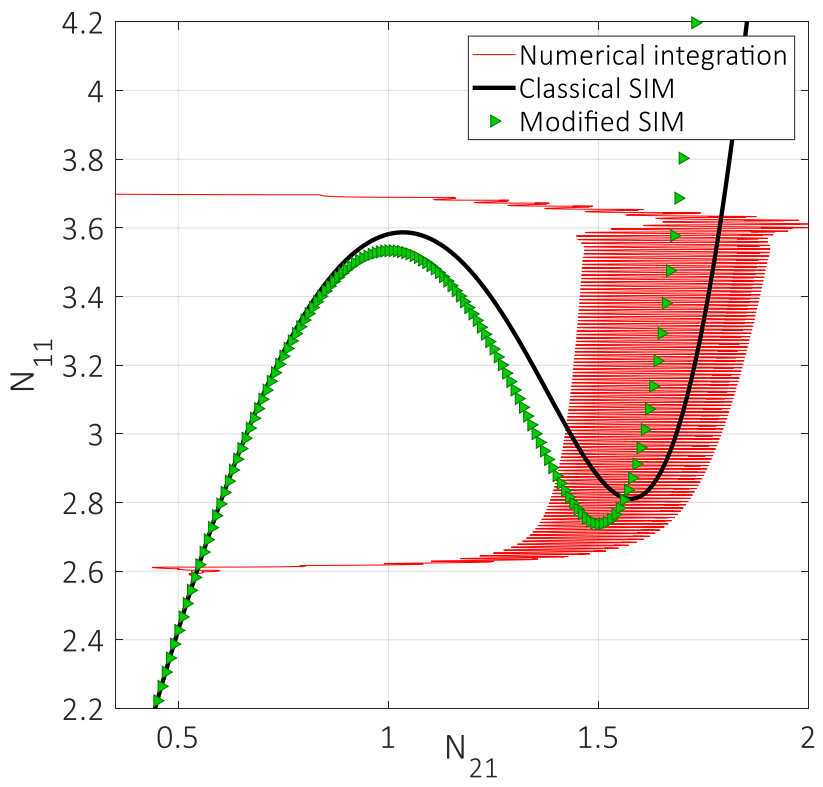

Fig. 4 A comparison between the analytical classical SIM (black line), the new one obtained by taking into consideration the third harmonics (green triangles and the numerical integration of the scaled system (2) (red curve).

with

$$
\left\{\begin{array}{l}
u_{0}=\frac{1}{2}\left(-q+\sqrt{-\frac{\Delta_{\text {card }}}{27}}\right) \\
v_{0}=\frac{1}{2}\left(-q-\sqrt{-\frac{\Delta_{\text {card }}}{27}}\right) .
\end{array}\right.
$$

Then, the modulus $N_{23}$ can be expressed as a function of $N_{21}$ as

$$
N_{23}=\mathscr{F}\left(N_{21}\right)=\left(\mathfrak{z}_{0}-\frac{1}{3} \frac{a_{2}}{a_{3}}\right)^{\frac{1}{2}} .
$$

Let us now investigate the dynamical behavior of the system at $\tau_{1}$ time scale. 
4.2 The system behavior at $\tau_{1}$ time scale

At slow time scale, equilibrium points around the SIM can be detected by treating the first equation of System (27) at the order $\varepsilon^{1}$ (See Equation (19)). It reads:

$$
\frac{\partial \varphi_{11}}{\partial \tau_{1}}=F_{1}\left(\varphi_{11}, \varphi_{11}^{*}, \varphi_{21}, \varphi_{21}^{*}\right)=-\left(\frac{\lambda}{2}+i \sigma\right) \varphi_{11}+\frac{\alpha}{2} \varphi_{21}+\frac{f}{2}
$$

Equation (49) can we written in the following system after writing it into its polar form

$$
\left\{\begin{array}{l}
\frac{\partial \delta_{11}}{\partial \tau_{1}}=f_{\delta 1}\left(\delta_{11}, N_{11}, \delta_{21}, N_{21}\right)=-\frac{\lambda}{2} N_{11}+\frac{\alpha}{2} N_{21} \cos \left(\delta_{21}-\delta_{11}\right)+\frac{f}{2} \cos \left(\delta_{11}\right) \\
\frac{\partial N_{11}}{\partial \tau_{1}}=f_{N 1}\left(\delta_{11}, N_{11}, \delta_{21}, N_{21}\right)=\frac{1}{N_{11}}\left(\frac{\alpha}{2} N_{21} \sin \left(\delta_{21}-\delta_{11}\right)-\sigma N_{11}-\frac{f}{2} \sin \left(\delta_{11}\right)\right) .
\end{array}\right.
$$

According to the analytical developments extended in Section 3.1.2, equilibrium points and singularities are described by the following matrix equation

$$
\underbrace{\left(\begin{array}{llll}
\frac{\partial h_{r 1}}{\partial \delta_{21}} & \frac{\partial h_{r 1}}{\partial N_{21}} & \frac{\partial h_{r 1}}{\partial \delta_{23}} & \frac{\partial h_{r 1}}{\partial N_{23}} \\
\frac{\partial h_{i 1}}{\partial \delta_{21}} & \frac{\partial h_{i 1}}{\partial N_{21}} & \frac{\partial h_{i 1}}{\partial \delta_{23}} & \frac{\partial h_{r 1}}{\partial N_{23}} \\
\frac{\partial h_{r 3}}{\partial \delta_{21}} & \frac{\partial h_{r 3}}{\partial N_{21}} & \frac{\partial h_{r 3}}{\partial \delta_{23}} & \frac{\partial h_{r 3}}{\partial N_{23}} \\
\frac{\partial h_{i 3}}{\partial \delta_{21}} & \frac{\partial h_{i 3}}{\partial N_{21}} & \frac{\partial h_{i 3}}{\partial \delta_{23}} & \frac{\partial h_{i 3}}{\partial N_{23}}
\end{array}\right)}_{\mathscr{M}}\left(\begin{array}{c}
\frac{\partial \delta_{21}}{\partial \tau_{1}} \\
\frac{\partial N_{21}}{\partial \tau_{1}} \\
\frac{\partial \delta_{23}}{\partial \tau_{1}} \\
\frac{\partial N_{23}}{\partial \tau_{1}}
\end{array}\right)=-\left(\begin{array}{cc}
\frac{\partial h_{r 1}}{\partial \delta_{11}} & \frac{\partial h_{r 1}}{\partial N_{11}} \\
\frac{\partial h_{i 1}}{\partial \delta_{11}} & \frac{\partial h_{i 1}}{\partial N_{11}} \\
0 & 0 \\
0 & 0
\end{array}\right)\left(\begin{array}{c}
f_{\delta 1}\left(\delta_{11}, N_{11}, \delta_{21}, N_{21}\right) \\
f_{N 1}\left(\delta_{11}, N_{11}, \delta_{21}, N_{21}\right)
\end{array}\right)
$$

Equilibrium points of the system, can be obtained by solving the following system

$$
\left\{\begin{array}{l}
\mathscr{H}=0 \\
f_{\delta 1}=f_{N 1}=0 \\
\operatorname{det}(\mathscr{M}) \neq 0
\end{array}\right.
$$

and singularities are reached when

$$
\left\{\begin{array}{l}
\mathscr{H}=0 \\
f_{\delta 1}=f_{N 1}=0 \\
\operatorname{det}(\mathscr{M})=0
\end{array}\right.
$$

\subsection{Results and Discussion}

Solving the systems of Equations (52) and (53), the equilibrium and singularities of the system are illustrated in Fig. 5 as a function of the detuning parameter $\sigma$ for several values of the external forcing term $f$ : (a) first column with $f=0.2$ (respectively (b) second column with $f=0.6$ and (c) third column with $f=0.65$ ). The amplitudes of $N_{11}, N_{21}$ and $N_{23}$ (down to top) are plotted versus the detuning parameter $\sigma$. It can be seen that the influence of the third harmonic is increasing with the amplitude of the external forcing $f$. So it is clear that the third harmonic has to be taken into account for higher values of $f$.

In Figure 6, for $\eta=\alpha=0.1$, the amplitudes of $N_{21}$ and $N_{11}$ are plotted versus the forcing term $f$ values between 0 and 1 at the resonance frequency $\omega=\omega_{0}(\sigma=0)$. Clearly the critical values of $f$ corresponding to the transition between 1 and 3 solutions depends on the number of harmonics in the analytical approach. In order to highlight the importance of generalizing the analytical approach by including higher harmonics, the following section is dedicated to validate the performance and reliability of the results obtained. 

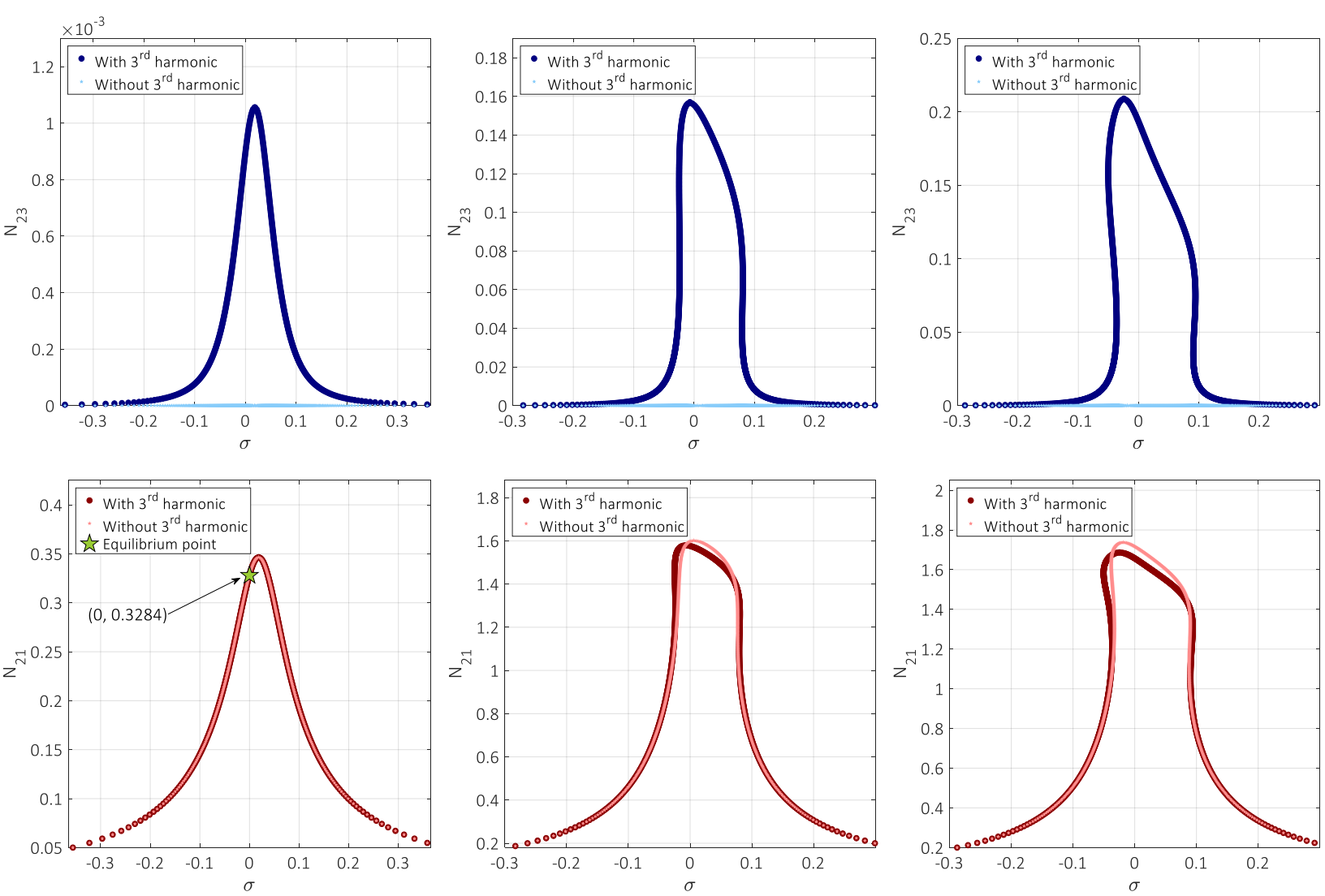

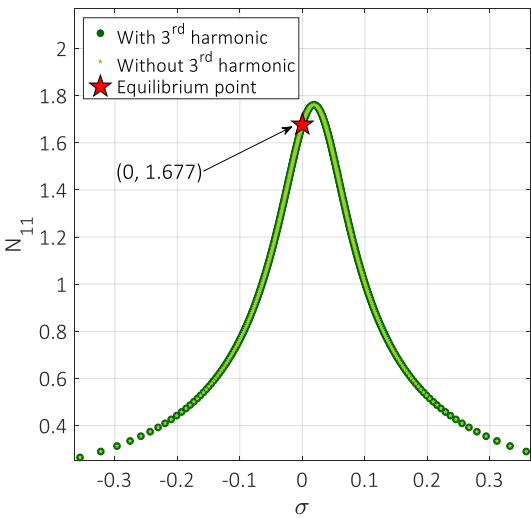

(a) $f=0.2$

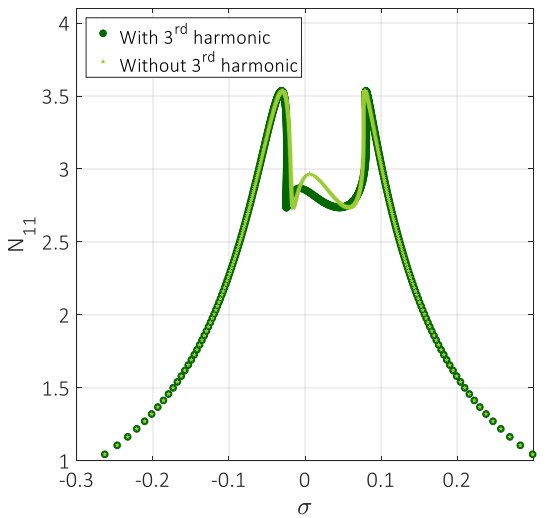

(b) $f=0.6$

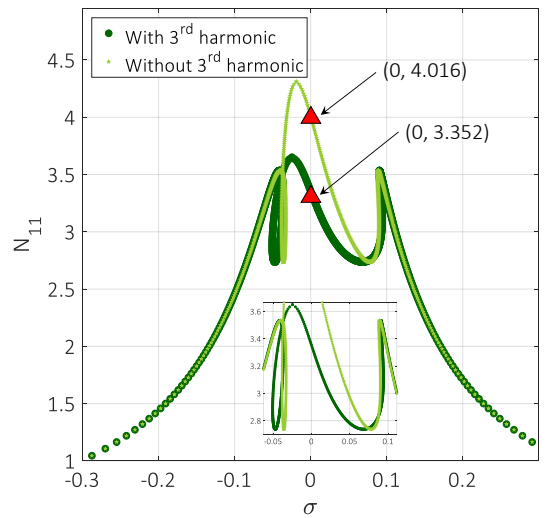

(c) $f=0.65$

Fig. 5 Variation of $N_{11}, N_{21}$ and $N_{23}$ versus the detuning parameter $\sigma$. (a) first column with $f=0.2$, (b) second column with $f=0.6$ and (c) third column with $f=0.65$.

\subsubsection{Response under weak forcing amplitude: $f=0.2$}

As a first example of validation between the analytical predictions and the numerical simulations, we consider the weak forcing term $f=0.2$. The analytical predictions of energy levels of both primary system and the NES via inclusion (or not) of the third harmonics are represented in Fig. 5 (a). At the resonance frequency $\omega_{0}$ (for $\sigma=0$ ), the corresponding numerical results obtained by solving System (2) using a Runge-Kutta algorithm compared to the SIM are depicted in Figure 7. For the initial conditions corresponding to $x(0)=V_{c}(0)=$ $V_{c}^{\prime}(0)=0$ and $x^{\prime}(0)=3.7$, the numerical integration of the scaled system oscillates around the upper branch of the new SIM. Once reaching the stability border, it jumps to follow the low level SIM amplitude to be finally be attracted by an equilibrium point represented with a pink triangle. 

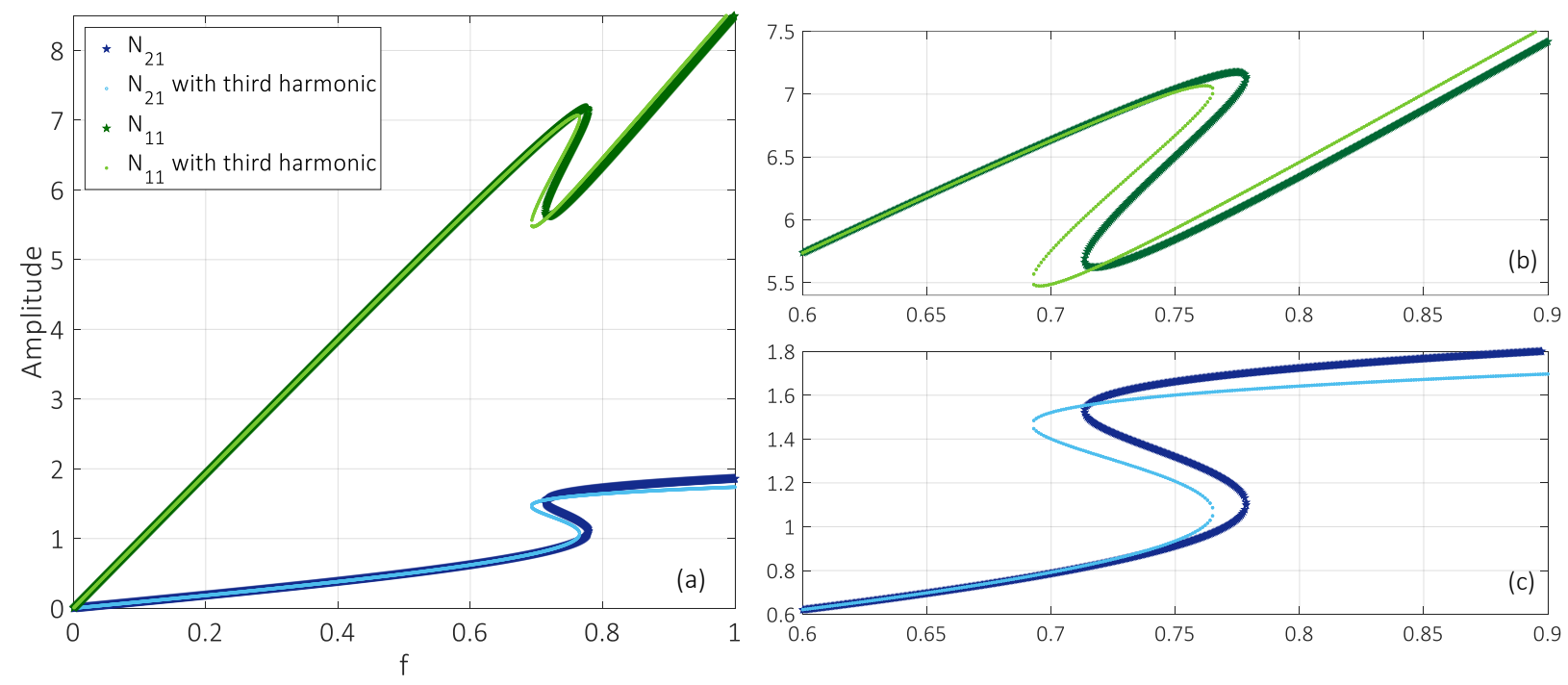

Fig. 6 (a) Variation of $N_{11}$ and $N_{21}$ versus the forcing term $f$ at the resonance frequency $\omega_{0}=\omega(\sigma=0)$, taking into consideration the presence and absence of the third harmonic. Zoom of $N_{11}$ (b) and $N_{21}$ versus $f$.

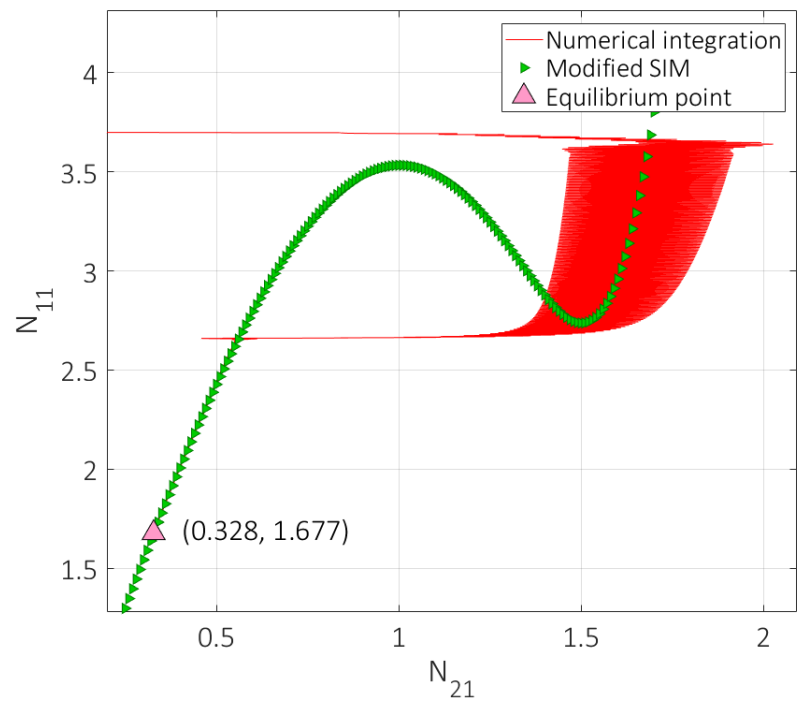

Fig. 7 SIM of the system with the corresponding numerical integration with the external forcing term $f=0.2$ and the detuning parameter $\sigma=0$.

The presence of an equilibrium point $\left(N_{21}, N_{11}\right)=(0.328,1.677)$ in Figure 7 indicates the fact that the system undergoes a periodic regime. This assumption is verified in Figure 8 where the velocity of the main system presents a periodic oscillations in the permanent regime with the amplitude 1.677. In order to confirm the previous analytical predictions presented in Figure 5 (a), we plot in Figure 9 the amplitude histories of $\left|\frac{d x}{d T}+i \Omega x\right|$ which corresponds to $N_{11}$ and $\left|\frac{d V_{c}}{d T}+i \Omega V_{c}\right|$ at the resonance frequency which corresponds to $\sigma=0$.

Numerical results are in good agreement with the analytical predictions depicted in Figure 5 (a) confirming the attraction of the system by an equilibrium point with amplitudes $N_{11}=1.677$ and $N_{21}=0.328$. Then, the NES is able to control the primary system against periodic external forces by presenting small amplitudes during periodic regimes.

\subsubsection{Response under higher forcing amplitude: $f=0.6$}

Figure 10 represents the analytical predictions of the different scenarios that the system amplitudes may reach according to the detuning parameter for the system with $f=0.6$. The system may present different possible 


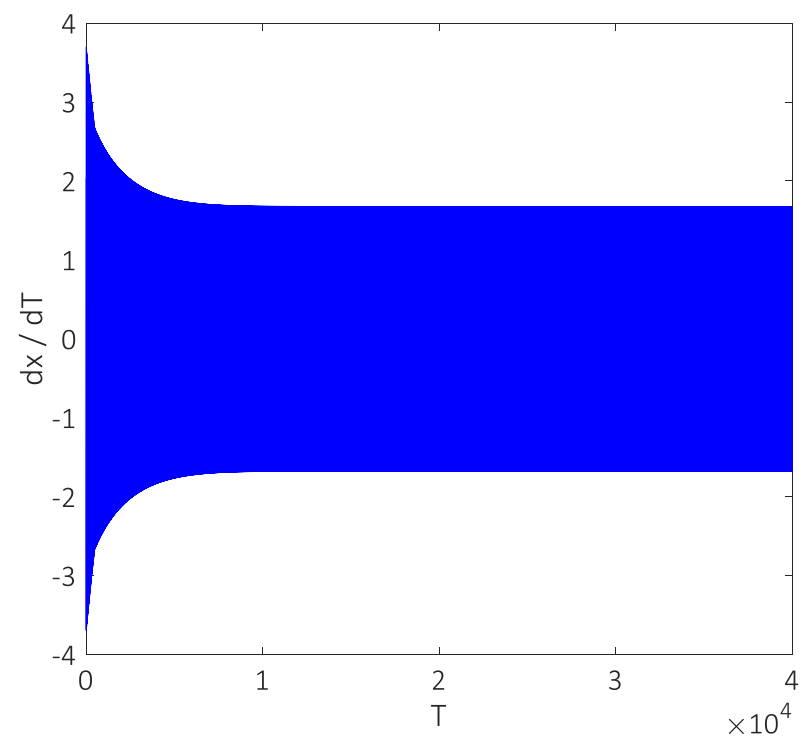

Fig. 8 Histories of velocity $d x / d T$ obtained by a direct integration of system (2) for the external forcing term $f=0.2$ and the detuning parameter $\sigma=0$
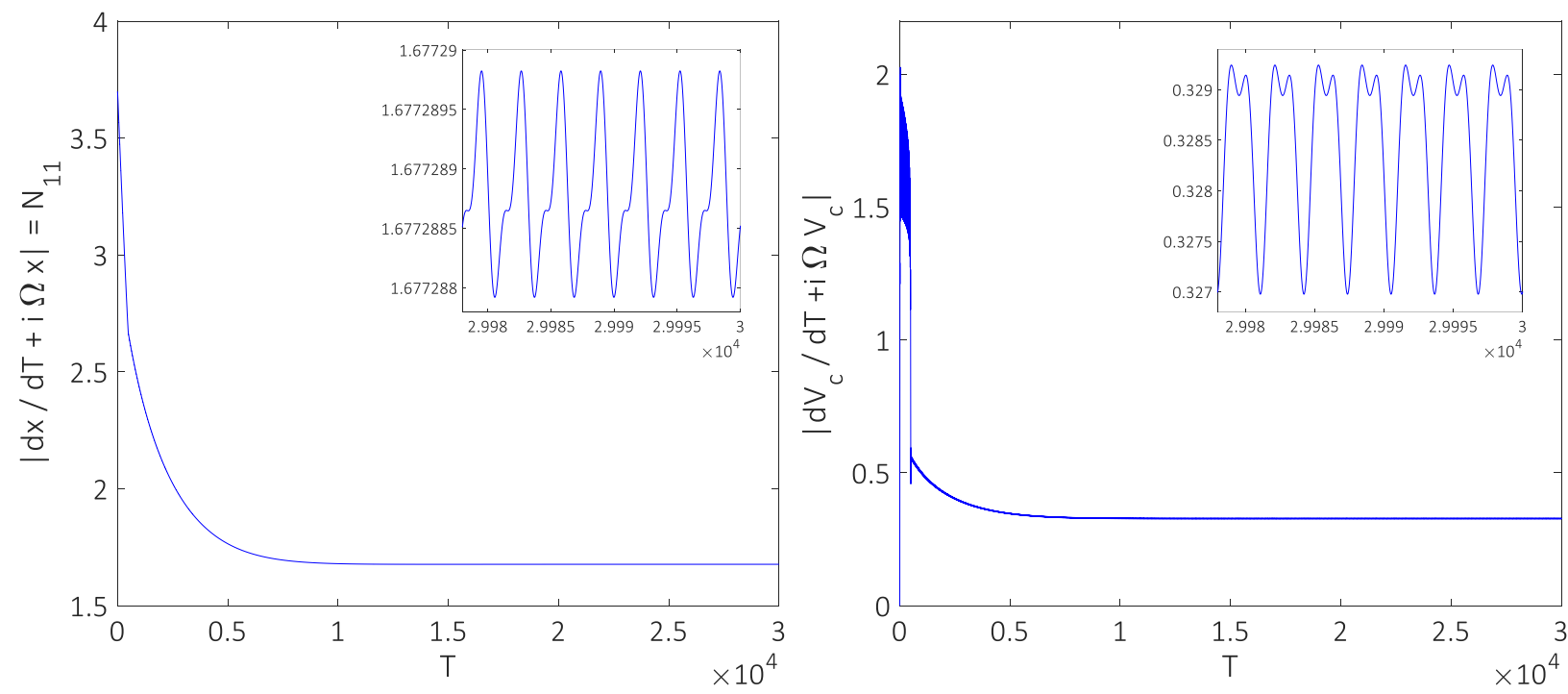

Fig. 9 Histories of system amplitudes $\left|\frac{d x}{d T}+i \Omega x\right|$ and $\left|\frac{d V_{c}}{d T}+i \Omega V_{c}\right|$ obtained by direct integration of the scaled system (2) with the external forcing term $f=0.2$ and the detuning parameter $\sigma=0$.

scenarios between periodic and quasi-periodic regimes which can be stable or unstable. Systems (52) and (53) allow an analytical identification of the dynamical regime to be reached. For instance, for $\sigma=0.0453$ System (53) is validated, with $N_{21}=1.5027$ and $N_{11}=2.7368$ which corresponds to the minimum value of the SIM. This later proves the existence of a singularity where the system is subjected to an SMR [38]. This prediction is validated in Figure 11, where we plot the numerical integration of System (2) compared to the SIM. Particularly, the system presents a closed loop with direct and reverse jumps phenomena between the stability borders $\left(\hat{n}_{11}, \hat{n}_{22}\right)$ and $\left(\hat{n}_{12}, \hat{n}_{21}\right)$.

Histories of the velocity $d x / d T$ illustrated in Figure 12 confirms the analogy between the analytical identification of SMR. Histories of the system amplitudes $\left|\frac{d x}{d T}+i \Omega x\right|$ and $\left|\frac{d V_{c}}{d T}+i \Omega V_{c}\right|$ are shown in Figure 13 confirming the analytical predictions illustrated in Figure 10 showing the fact that the system hits two different points during long time span. 


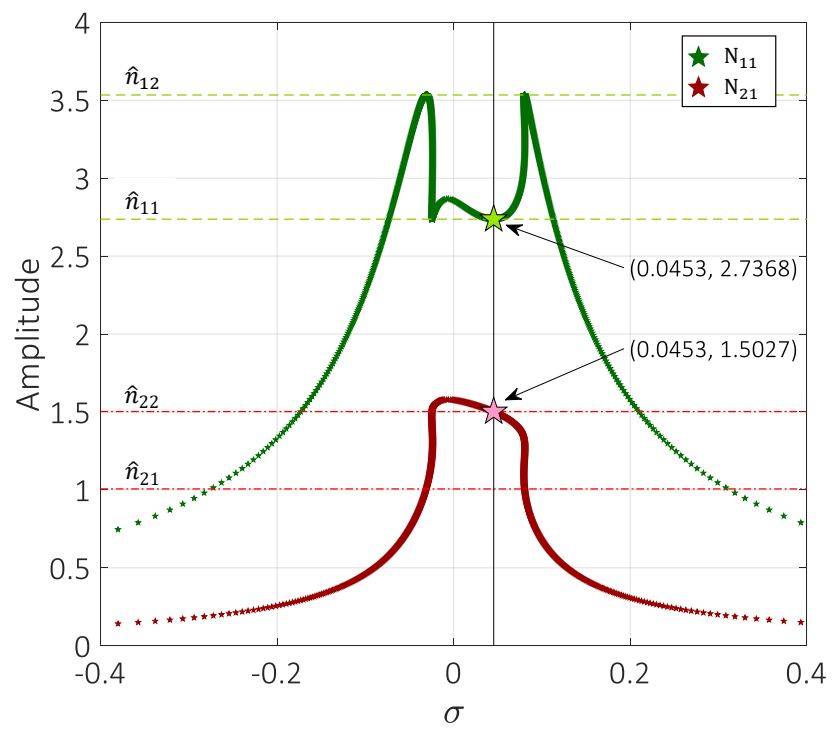

Fig. 10 Variation of energy of both the main system and the NES with respect to the detuning parameter $\sigma$ for the external forcing term $f=0.6$.

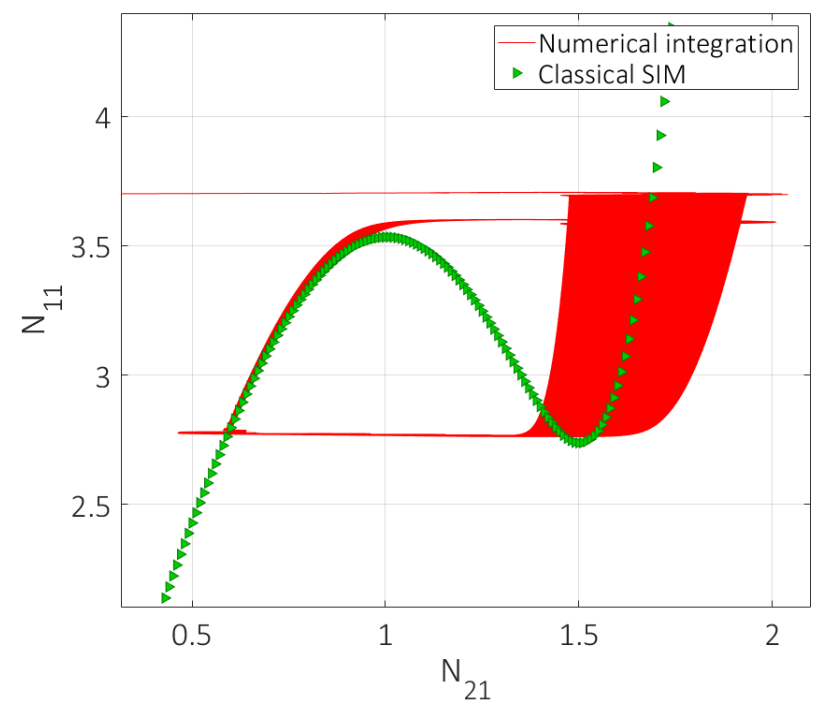

Fig. 11 SIM of the system with the corresponding numerical integration with the external forcing term $f=0.6$ and the detuning parameter $\sigma=0.0453$.

\subsubsection{Response under forcing term $f=0.65$}

For a higher forcing term with $f=0.65$, the analytical predictions of energy levels of both primary system and the NES via inclusion (or not) the third harmonics are represented in Fig. 5 (c). For the detuning parameter $\sigma=0$ which corresponds to the resonance frequency $\Omega=\omega_{0}$. It is seen that the inclusion of third harmonics in the system changes the curve corresponding to consideration of only first harmonic dramatically. Solving the scaled system (2) numerically, we plot in Fig. 14 the histories of variables $\left|\frac{d x}{d T}+i \Omega x\right|$ which corresponds to $N_{11}$ and $\left|\frac{d V_{c}}{d T}+i \Omega V_{c}\right|$. Noticeably, the numerical integration of the system for $\sigma=0$ indicates that the value of $N_{1} 1$ corresponds to 3.2224 , which is in good agreement with the analytical prediction represented with a magenta triangle in Fig. 5 (c) when including the effects of the third harmonic. To conclude, the consideration of the third harmonic in the analytical developments permits to predict the system behavior closer to the exact behavior obtained by numerical integration, especially for higher forcing terms. 


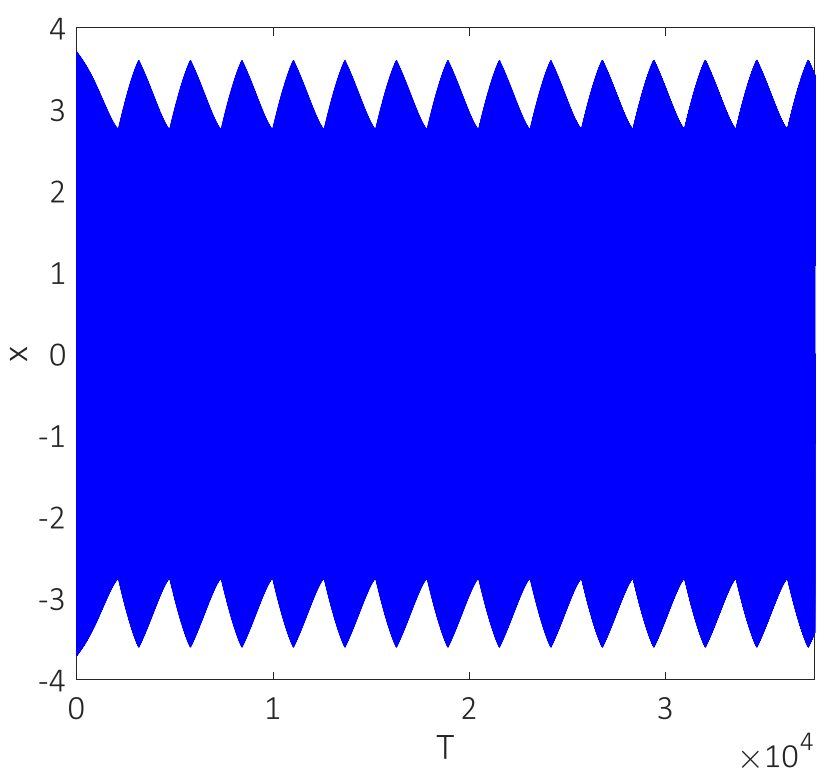

Fig. 12 Histories of velocity $d x / d T$ obtained by a direct integration of system (2) for the external forcing term $f=0.6$ and the detuning parameter $\sigma=0.0453$
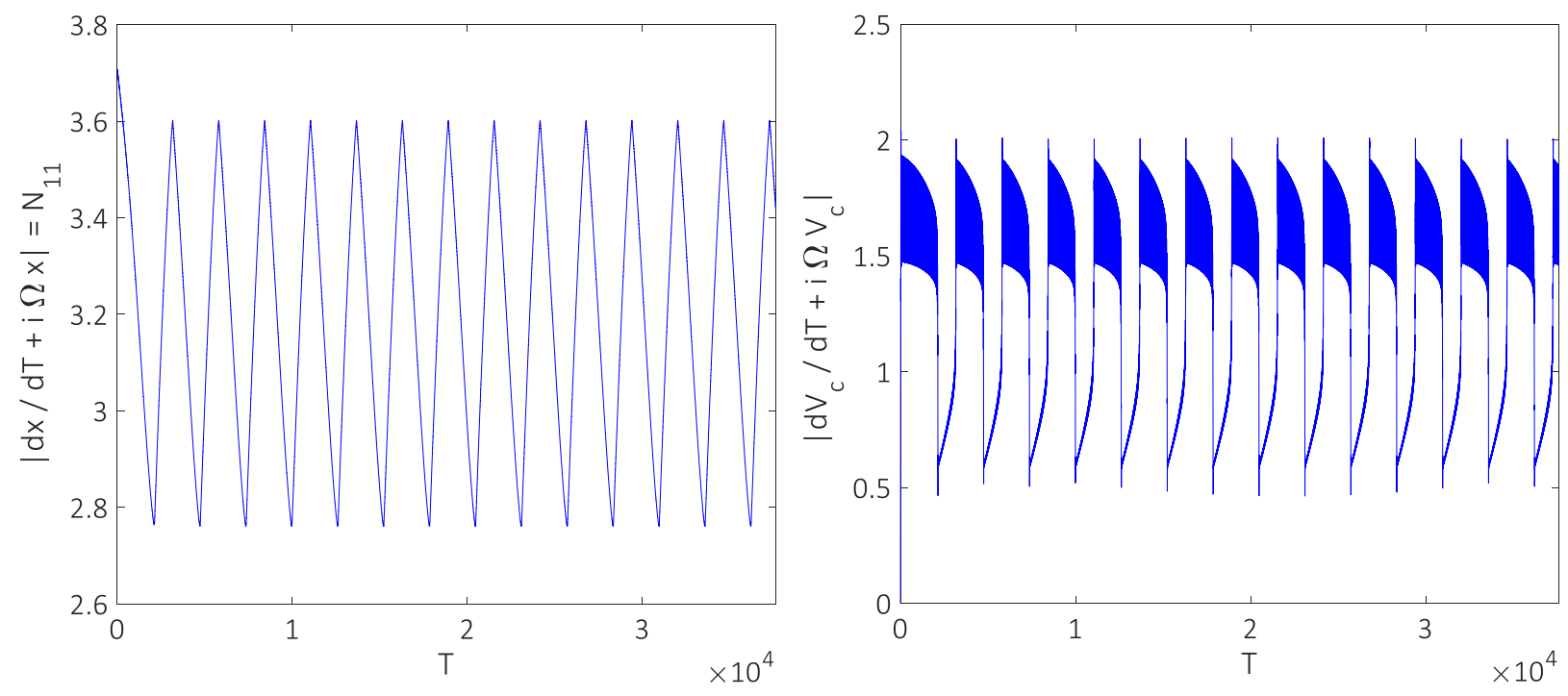

Fig. 13 Histories of system amplitudes $\frac{d x}{d T}+i \Omega x$ and $\frac{d V_{c}}{d T}+i \Omega V_{c}$ obtained by direct integration of the scaled system (2) with the external forcing term $f=0.6$ and the detuning parameter $\sigma=0.0453$.

\section{Conclusions}

An extension of complex variables of Manevitch is applied to a scaled general two degrees of freedom system for taking into account the effects of higher harmonics. The system consists of a primary structure coupled to a nonlinear energy sink. The problem is treated analytically around the 1:1 resonance by using a multiple scale approach. The general slow invariant manifold of the system including all harmonics is obtained at fast time scale. Studying the dynamics of the system at slow time around its slow invariant manifold permits the detection of equilibrium and singular points. These points correspond to periodic and modulated regimes, respectively. This approach is then applied to a system consisting of an electroacoustic loudspeaker linearly coupled to an electric nonlinear shunt circuit acting as a nonlinear energy sink. In this paper we show that it is necessary to take into consideration the presence of the third harmonic in analytical developments. Comparisons between results obtained from keeping first harmonics and then keeping first and third harmonics are carried out, 

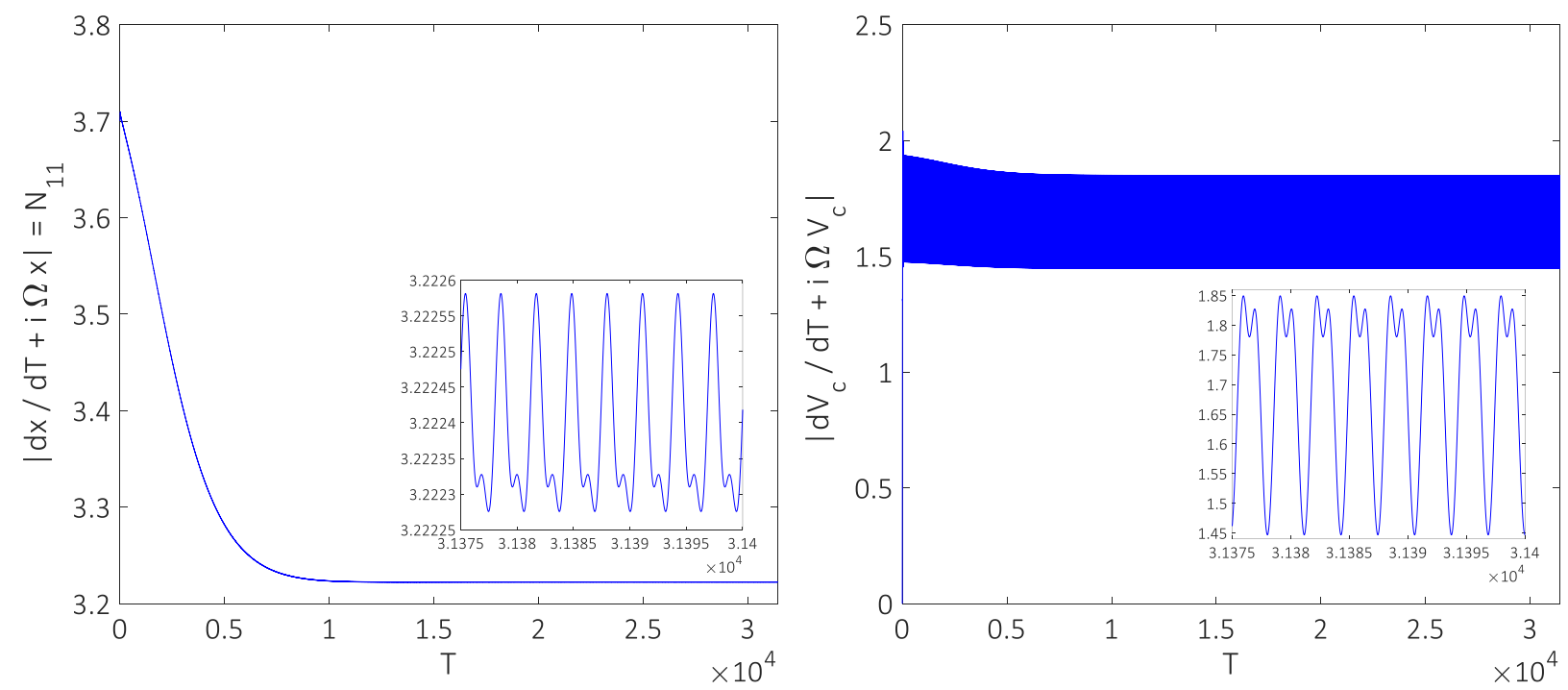

Fig. 14 Histories of system amplitudes $\left|\frac{d x}{d T}+i \Omega x\right|$ and $\left|\frac{d V_{c}}{d T}+i \Omega V_{c}\right|$ obtained by direct integration of the scaled system (2) with the external forcing term $f=0.65$ and the detuning parameter $\sigma=0$.

highlighting the importance of employing the higher harmonic in the analytical approaches and so in prediction of more punctual positions of equilibrium and singular points. Good agreements between results obtained from direct numerical integration of the system equations and those obtained from analytical predictions are seen. Future works are devoted to the physical setup of an electroacoustic loudspeaker confirming the obtained numerical integration and analytical predictions, in addition to an optimization process for better design of the nonlinear energy sink.

\section{Acknowledgements}

This work was conducted in the framework of the LABEX CELYA (ANR-10-LABX-0060) of the "Université de Lyon" within the program "Investissement d'Avenir" (ANR-11-IDEX-0007) operated by the French National Research Agency (ANR).

\section{Compliance with ethical standards}

Conflict of interest The authors declare no conflict of interest.

\section{References}

1. G. W. Housner, L. A. Bergman, T. K. Caughey, A. G. Chassiakos, R. O. Claus, S. F. Masri, R. E. Skelton, T. T. Soong, B. F. Spencer, and J. T. P. Yao. Structural control: past, present and future. J Eng Mech., 123:897-971, 1997.

2. S. Korkmaz. A review of active structural control: challenges for engineering informatics. Comput Struct, 89:2113-2132, 2011.

3. D. J. Mead. Passive vibration control. Wiley, West Sussex, England, 1999.

4. R.A. Ibrahim. Recent advances in nonlinear passive vibration isolators. Journal of Sound and Vibration, 314(3):371 - 452, 2008.

5. H. Frahm. Device for damping vibrations of bodies, 1909. U.S. Patent No. 989958.

6. H. V. Helmholtz. Die lehre von den tonempfindungen als physiologische grundlagefur die theorie der musik. Brunswick, Druck und Verlag von Friedrich Vieweg und Sons, 1863.

7. A. H. Nayfeh and B. Balachandran. Modal Interactions in Dynamical and Structural Systems. Applied Mechanics Reviews, 42(11S):S175-S201, 1989.

8. M.I. Younis. MEMS Linear and Nonlinear Statics and Dynamics. Springer, 2011

9. R. E. Roberson. Synthesis of a nonlinear dynamic vibration absorber. J. Franklin Inst., 254:205-220, 1952.

10. G. Gatti and M. J. Brennan. On the effects of system parameters on the response of a harmonically excited system consisting of weakly coupled nonlinear and linear oscillators. Journal of Sound and Vibration, 330(18):4538 - 4550, 2011. 
11. E. Sevin. On the parametric excitation of pendulum-type vibration absorber. Journal of Applied Mechanics, 28(3):330-334, 1961.

12. R. S. Haxton and A. D. S. Barr. The autoparametric vibration absorber. Journal of Engineering for Industry, 94(1):119-125, 1972 .

13. J. B. Hunt and J.-C. Nissen. The broadband dynamic vibration absorber. Journal of Sound and Vibration, 83(4):573-578, 1982.

14. S. Ema and E. Marui. Damping characteristics of an impact damper and its application. International Journal of Machine Tools and Manufacture, 36(3):293-306, 1996.

15. A. F. Vakakis, O. V. Gendelman, L. A. Bergman, D. M. McFarland, G. Kerschen, and Y. S. Lee. Nonlinear Targeted Energy Transfer in Mechanical and Structural Systems. Solid Mechanics and Its Applications. Springer Netherlands, 2008.

16. A. F. Vakakis. Inducing passive nonlinear energy sinks in vibrating systems. Journal of Vibration and Acoustics, $123(3): 324$ $-332,2001$.

17. O. Gendelman, L. I. Manevitch, A. F. Vakakis, and R. MCloskey. Energy pumping in nonlinear mechanical oscillators: Part idynamics of the underlying hamiltonian systems. Journal of Applied Mechanics, 68(1):34-41, 2001.

18. Y. S. Lee, A. F. Vakakis, L. A. Bergman, D. M. McFarland, and G. Kerschen. Enhancing robustness of instability suppression by means of multi-degree-of-freedom nonlinear energy sinks. AIAA Journal, 46(18):1371-1394, 2008.

19. N. E. Wierschem, S. A. Hubbard, J. Luo, L. A. Fahnestock, B. F. Spencer Jr., M. D. McFarland, Quinn D. D., A.F. Vakakis, and L.A. Bergman. Response attenuation in a large-scale structure subjected to blast excitation utilizing a system of essentially nonlinear vibration absorbers. Journal of Sound and Vibration, 389:52-72, 2017.

20. M. Weiss, B. Vaurigaud, A. Ture Savadkoohi, and C.-H. Lamarque. Control of vertical oscillations of a cable by a piecewise linear absorber. Journal of Sound and Vibration, 435:281-300, 2018.

21. B. Cochelin, P. Herzog, and P.-O. Mattei. Experimental evidence of energy pumping in acoustics. Comptes Rendus Mcanique, 334(11):639 - 644, 2006.

22. L. I. Manevitch. The description of localized normal modes in a chain of nonlinear coupled oscillators using complex variables. Nonlinear Dynamics, 25(1):95-109, Jul 2001.

23. O. V. Gendelman. Targeted energy transfer in systems with non-polynomial nonlinearity. Journal of Sound and Vibration, $315(3): 732-745,2008$.

24. A. Ture Savadkoohi, C.-H. Lamarque, and Z. Dimitrijevic. Vibratory energy exchange between a linear and a nonsmooth system in the presence of the gravity. Nonlinear Dynamics, 70(2):1473-1483, Oct 2012.

25. M. Weiss, M. Chenia, A. Ture Savadkoohi, C.-H. Lamarque, B. Vaurigaud, and A. Hammouda. Multi-scale energy exchanges between an elasto-plastic oscillator and a light nonsmooth system with external pre-stress. Nonlinear Dynamics, 83(1):109135, Jan 2016.

26. C.-H. Lamarque, A. Ture Savadkoohi, S. Charlemagne, and P. Abdoulhadi. Nonlinear vibratory interactions between a linear and a nonsmooth forced oscillator in the gravitational field. Mechanical Systems and Signal Processing, 89:131-148, 2017.

27. D. Bitar, A. Ture Savadkoohi, C.-H. Lamarque, E. Gourdon, and M. Collet. Targeted nonlinear energy transfer for electroacoustic absorbers. In Stefano Lenci and Ivana Kovacic, editors, IUTAM Symposium on Exploiting Nonlinear Dynamics for Engineering Systems. Springer, 2019.

28. D. Bitar, E. Gourdon, C.-H. Lamarque, and M. Collet. Shunt loudspeaker using nonlinear energy sink. Journal of Sound and Vibration, 456:254-271, 2019.

29. C.-H. Lamarque, O. V. Gendelman, A. Ture Savadkoohi, and E. Etcheverria. Targeted energy transfer in mechanical systems by means of non-smooth nonlinear energy sink. Acta Mechanica, 221(1):175, May 2011.

30. O. V. Gendelman. Targeted energy transfer in systems with external and self-excitation. Proceedings of the Institution of Mechanical Engineers, Part C: Journal of Mechanical Engineering Science, 225(9):2007-2043, 2011.

31. A. H. Nayfeh and D. T. Mook. Nonlinear Oscillations. Wiley Classics Library. Wiley, 2008.

32. J. Kevorkian and J. D. Cole. The Method of Multiple Scales for Ordinary Differential Equations, pages 267-409. Springer New York, New York, NY, 1996.

33. F. Verhulst. Singular perturbation methods for slow-fast dynamics. Nonlinear Dynamics, 50(4):747-753, 2007.

34. Lamberto Cesari. Functional analysis and galerkin's method. The Michigan Mathematical Journal, $11(4): 385-414,1964$.

35. M. Urabe. Galerkin's procedure for nonlinear periodic systems. Archive for Rational Mechanics and Analysis, 20(2):120$152,1965$.

36. A. Ture Savadkoohi, C.-H. Lamarque, and M. V. Contessa. Trapping vibratory energy of main linear structures by coupling light systems with geometrical and material non-linearities. International Journal of Non-Linear Mechanics, 80:3 - 13, 2016. Dynamics, Stability, and Control of Flexible Structures.

37. L. Shampine and M. Reichelt. The matlab ode suite. SIAM Journal on Scientific Computing, 18(1):1-22, 1997.

38. Y. Starosvetsky and O. V. Gendelman. Strongly modulated response in forced 2dof oscillatory system with essential mass and potential asymmetry. Physica D: Nonlinear Phenomena, 237(13):1719 - 1733, 2008. 